Disponível em

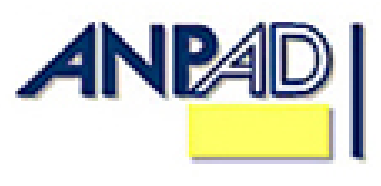

http://www.anpad.org.br/rac

RAC, Curitiba, v. 15, n. 4, art. 2,

pp. 580-607, Jul./Ago. 2011

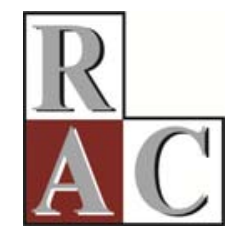

\title{
O Conceito Desempenho em Estudos Organizacionais e Estratégia: um Modelo de Análise Multidimensional
}

\section{The Concept of Performance on Organizational Studies and Strategy: a Multi- Dimensional Model of Analysis}

Queila Regina Souza Matitz *

E-mail: queila.matitz@gmail.com

Universidade Positivo - UP

Curitiba, PR, Brasil.

Sergio Bulgacov

E-mail: s.bulgacov@ufpr.br Universidade Federal do Paraná - UFPR/DAGA

Curitiba, PR, Brasil.

* Endereço: Queila Regina Souza Matitz

UP, Rua Prof. Pedro Viriato Parigot de Souza, 5300, Campo Comprido, Curitiba/PR, 81280-330.

Copyright (C) 2011 RAC. Todos os direitos, até mesmo de tradução, são reservados. É permitido citar parte de artigos sem autorização prévia, desde que seja identificada a fonte. 


\title{
Resumo
}

O presente trabalho propõe um Modelo Multidimensional de Análise do Conceito Desempenho Organizacional, desenvolvido com base em modelos anteriores e utilizado para a realização de análise de conteúdo comparativa, categorial, transnacional, longitudinal, descritivo-quantitativa e inferencial-qualitativa de pesquisas empíricas brasileiras e estrangeiras das áreas de estudos de organizações e de estratégia, publicadas entre 2005 e 2007. Conclui-se que o novo modelo ajustou-se à análise de pesquisas empíricas, além de ampliar modelos anteriores em termos de exaustividade e exclusividade das categorias de análise. Em particular, o novo modelo constitui um avanço ao incluir categorias de análise relacionadas aos padrões de atribuição e de interação causal. A análise comparativa das pesquisas brasileiras e estrangeiras revelou o seguinte: (a) preferência pelo uso de indicadores unidimensionais de desempenho, das áreas econômico-financeiro-mercadológica e a partir de perspectiva teórica racional; (b) preferência pelo uso de dados objetivos secundários do nível de análise organizacional e perspectiva temporal estática não-cumulativa comparativa retrospectiva; e (c) preferência por desenhos de pesquisa que pressupõem relações multilineares diretas e horizontais, em que o desempenho funciona como variável dependente.

Palavras-chave: desempenho organizacional; modelo multidimensional de análise; estratégia; estudos organizacionais; análise de conteúdo.

\begin{abstract}
A Multi-dimensional Analytical Model of Organizational Performance Concept, developed on the basis of previous models, was used to accomplish a comparative, categorical, cross-national, longitudinal, descriptivequantitative and inferential-qualitative content-analysis of Brazilian and Foreign empirical studies from Organizational Studies and Strategy areas, published from 2005 to 2007. It was concluded that this new model applies to empirical research analysis, being distinguished from previous models in terms of greater exaustivity and exclusivity of its categories of analysis. Particularly, the new model constitutes an advance because it includes categories of analysis related to attribution patterns and causal interaction. Comparative analysis of Brazilian and foreign researches revealed (a) preference for unidimensional performance indicators from economic-financial-marketing areas and from a rational theoretical perspective; (b) preference for secondary and objective data, from organizational level of analysis and not-cumulative comparative retrospective static temporal perspective; and (c) preference for research designs that estimate direct and horizontal multilinear relations between the variables, in which performance is used as a dependent variable.
\end{abstract}

Key words: organizational performance; multi-dimensional analytical model; strategy; organizational studies; content analysis. 


\section{Introdução}

Considerando-se a importância teórica, empírica e prática do conceito de desempenho, um grupo ainda reduzido de pesquisadores tem-se dedicado ao estudo sistemático da natureza deste construto, como fundamento para o desenvolvimento conceitual do tema e para a superação de limitações historicamente observadas, em termos de operacionalização, mensuração e comparação de pesquisas empíricas. Propõe-se aqui, portanto, como forma de contribuir para ampliar a compreensão da natureza do conceito de desempenho nos campos de estudos de organizações e de estratégia: (a) um Modelo Multidimensional de Análise do Conceito Desempenho e (b) uma análise comparativa de pesquisas empíricas brasileiras e estrangeiras com base no uso das dimensões, propriedades e atributos propostos no modelo.

Um Modelo Multidimensional de Análise é um recurso de apoio à representação de conceitos complexos, sob o ponto de vista conceitual e operacional. A principal utilidade deste tipo de modelo é a possibilidade de visualização das diversas alternativas - bem como suas respectivas vantagens e desvantagens - disponíveis para o pesquisador, cujo objetivo é a conceituação e mensuração de determinado conceito. O modelo aqui proposto pretende servir como guia para a representação descritiva das múltiplas dimensões do conceito de desempenho, considerados seus atributos conceituais e operacionais. Desse modo, pretende-se auxiliar pesquisadores das áreas de organizações e de estratégia a identificarem atributos relacionados à conceituação e à operacionalização - formal e relacional - do conceito de desempenho.

Justifica-se a importância do presente trabalho: (a) em função da necessidade de aperfeiçoamento constante na conceituação, operacionalização e mensuração dos fenômenos centrais estudados pelas áreas de estudos de organizações e de estratégia e (b) em função da necessidade de superar a falta de exclusividade e exaustividade das categorias propostas por esquemas de classificação multidimensional do conceito de desempenho propostos anteriormente. Justifica-se o trabalho, ainda, com base na importância da realização de revisões e meta-análises de pesquisas empíricas de modo a desvendar definições e operacionalizações que têm sido utilizadas por estudiosos dessas áreas de estudos.

\section{A importância e os desafios do uso do conceito desempenho nos campos de estudos organizacionais e de estratégia}

Cameron e Whetten (1983), em obra clássica a respeito dos modelos de efetividade organizacional, chamam a atenção para a importância da noção de desempenho para os estudos organizacionais. Como lembram os autores, o construto da efetividade organizacional apresenta implicações: (a) teóricas, pois se encontra no centro de todos os modelos organizacionais; (b) empíricas, pois é a variável dependente final em pesquisa organizacional; e (c) práticas, pois há necessidade de avaliar os resultados obtidos pelas organizações.

Em trabalho contemporâneo ao de Cameron e Whetten (1983), Lewin e Minton (1986) afirmam que a questão da efetividade organizacional tem servido como tema unificador por mais de um século de pesquisa em administração e design organizacional, embora a pesquisa empírica não tenha conseguido desenvolver uma teoria universal de efetividade organizacional. Mais recentemente, Richard, Devinney, Yip e Johnson (2009) retomam o debate a respeito da necessidade de discussão a respeito do significado e dos métodos de mensuração do desempenho utilizados por pesquisadores da área da administração. Segundo os autores, a natureza multidimensional do conceito de desempenho deve ser compreendida e devidamente considerada para evitar o uso de medidas de pouca cobertura em relação à amplitude e profundidade do construto. 


\section{Propostas recentes de classificação conceitual das dimensões do conceito desempenho organizacional}

Historicamente, a multidimensionalidade do conceito de desempenho tem sido reconhecida como uma de suas principais características. E, enquanto diferentes grupos de autores têm buscado desenvolver medidas agregadas de desempenho organizacional e/ou modelos de avaliação de efetividade organizacional, outro grupo tem-se dedicado ao estudo da natureza multidimensional do conceito. Estudos dessa natureza são importantes, na medida em que esclarecem quais são os atributos ou características do conceito a serem levados em consideração por pesquisadores.

Uma busca preliminar nas principais bases de dados dos periódicos das áreas de organizações e de estratégia demonstrou que estudos recentes, a respeito da natureza multidimensional do conceito de desempenho organizacional, são raros na literatura das áreas de organizações e de estratégia. Uma segunda busca, com base na identificação das referências utilizadas por artigos que abordam o assunto - a natureza multidimensional do conceito de desempenho organizacional - possibilitou a identificação de trabalhos recentes de classificação, entre os quais Thach e Axinn (1994), Matthyssens e Pauwels (1996), Madsen (1998), Katsikeas, Leonidou e Morgan (2000), Carneiro, Silva, Rocha e Hemais (2005) e Richard et al. (2009). Os três últimos trabalhos foram selecionados por serem derivações dos modelos anteriores e avaliados com base nos critérios de classificação taxonômica e tipológica propostos por Bailey (1994).

Conforme Bailey (1994), a adequação de uma classificação está diretamente relacionada à adequação das dimensões ou variáveis sobre as quais é realizada e, portanto, a identificação das características fundamentais do fenômeno a ser classificado constitui etapa essencial do processo. Quanto às regras básicas dos processos de classificação, incluem-se a exaustividade e a exclusividade. A exaustividade significa a inclusão de classes apropriadas para todos os objetos a serem classificados, enquanto a exclusividade pressupõe a existência de apenas uma classe correta para cada objeto, ou seja, o mesmo objeto não pode pertencer a duas classes ao mesmo tempo. Outras regras dos processos de classificação incluem: simplicidade de forma e conteúdo ou facilidade de compreensão; parcimônia ou redução da complexidade de fenômenos multidimensionais em níveis adequados ao desenvolvimento de descrições, análises e interpretações de dados; pertinência ou adequação do sistema de categorias quanto às intenções da investigação; objetividade e fidelidade ou facilidade de replicação da classificação, obtendo-se os mesmos resultados; e produtividade ou fertilidade do sistema de classificação, em termos de inferências, dados e geração de novas propostas de pesquisas.

Primeiramente, verificou-se que uma das principais áreas de estudo da natureza multidimensional do conceito de desempenho tem sido o campo de investigações de resultados de exportações no contexto do marketing internacional, objeto de estudo de Katsikeas et al. (2000). A partir da análise de estudos empíricos, os autores criticam o uso das medidas de desempenho encontradas e defendem a utilização da abordagem contingencial, como solução das dificuldades de consistência e conflito entre os resultados das pesquisas. Outros resultados da meta-análise incluem: (a) a constatação da predominância do uso do conceito de desempenho como variável dependente e (b) o desenvolvimento de um sistema de classificação do uso do conceito de desempenho na área de exportações. O sistema de classificação desenvolvido pelos autores é composto de três grandes dimensões: (a) operacionalização, que inclui o significado conferido ao desempenho (efetividade, eficiência ou adaptabilidade), o quadro de referência para avaliação do desempenho (desempenho no mercado doméstico, evolução no tempo, desempenho da indústria ou concretização das metas prédefinidas), a importância dos stakeholders na definição de indicadores de desempenho (se orientada internamente, com foco no competidor ou com foco no cliente) e o horizonte ou corte temporal considerado na mensuração (desempenho histórico, desempenho atual ou perspectiva de futuro); (b) amostragem, que inclui a unidade de análise (a corporação, o empreendimento de exportação ou determinado produto/linha de produtos) e o escopo ou nível de análise (todos os mercados de exportação, uma região geográfica ou um único país); (c) coleta de dados, que inclui a fonte dos dados (primários ou secundários) e o modo de abordagem (objetivo ou subjetivo). 
Embora algumas das propriedades sejam específicas do contexto dos estudos de desempenho de exportações, a classificação fornece pistas a respeito de elementos a serem considerados em quadro mais abrangente, aplicável a estudos de desempenho em outras áreas de estudos organizacionais, como, por exemplo, a separação entre aspectos relacionados à operacionalização - incluindo elementos relacionados ao significado atribuído ao conceito e a elementos condicionantes da forma de conceituação - e à amostragem e coleta de dados. Quanto à pertinência da classificação proposta, com base na descrição da metodologia utilizada pelos autores, não ficam claros os critérios utilizados; entretanto é possível inferir que houve tentativa de separação entre decisões relacionadas à base teórica - o que seria mensurado - e decisões metodológicas - como seria realizada a mensuração. Quanto à qualidade da classificação proposta, ressalta-se a restrição da classificação daqueles elementos encontrados efetivamente nas pesquisas analisadas pelos autores, desconsiderando-se a possibilidade de desdobramento das categorias de análise por meio da inclusão de outros atributos. Por exemplo, poderiam ter sido incluídos outros tipos de stakeholders, sob a categoria perspectiva dos stakeholders ou, ainda, poderia ter sido incluída uma categoria mista na classe fonte de coleta de dados.

Carneiro et al. (2005) também analisaram trabalhos anteriores de pesquisadores defensores do uso de múltiplas dimensões na conceituação do desempenho organizacional e destacam algumas contribuições de análises anteriores a respeito das vantagens e desvantagens do uso de: indicadores financeiros versus indicadores operacionais; fontes de dados primárias versus fontes de dados secundárias; ponto de vista externo versus ponto de vista interno e medidas retrospectivas versus medidas prospectivas. Quanto à forma de mensuração do desempenho em pesquisas empíricas, de acordo com os autores, se destacam três tipos: o uso de uma única medida diretamente observável, o uso de múltiplas medidas não agregadas e diretamente observáveis ou o uso de escalas compostas por meio da agregação de múltiplas medidas.

Com o objetivo de contribuir para o avanço de um esquema conceitual capaz de esclarecer algumas das principais dimensões do fenômeno desempenho, Carneiro et al. (2005) sintetizaram estudos anteriores e desenvolveram um esquema conceitual multidimensional. Similar à proposta desenvolvida por Katsikeas et al. (2000), o esquema de classificação de Carneiro et al. (2005) tem como propósito servir de guia para futuras pesquisas e como facilitador de esforços comparativos entre diferentes trabalhos. Metodologicamente, o esquema foi desenvolvido a partir de quadros analíticos, propostos anteriormente pela literatura e a partir da análise de conteúdo de 150 artigos das áreas de estratégia, marketing, finanças, empreendedorismo e negócios internacionais, cujo foco era 0 desempenho organizacional e temas relacionados. Justificou-se a importância de seu desenvolvimento com base em duas dificuldades encontradas por pesquisadores, ao utilizar o conceito de desempenho: (a) a definição conceitual e (b) a mensuração do fenômeno. No campo Dimensões Conceituais foram incluídas propriedades que dizem respeito à natureza do conceito de desempenho em relação às seguintes categorias: ponto de vista do stakeholder (acionistas, clientes, empregados, administradores, entre outros); classes de medidas (econômica, mercadológica, estratégica, social, entre outras); quadro de referência (absoluto ou relativo); e orientação temporal (estática ou dinâmica). No campo Dimensões Metodológicas foram incluídos: Unidade de Análise (país, indústria, empresa, divisões, empreendimento); Modo de Abordagem (objetivo ou subjetivo, a partir e fontes primárias ou secundárias) e Estrutura dos Indicadores (indicadores, independentes ou escalas compostas).

Quanto à avaliação crítica das dimensões propostas, pode-se destacar: (a) As dimensões Ponto de Vista do Stakeholder e Classes de Medidas apresentam sobreposição dos diferentes atributos propostos, os quais poderiam fazer parte de duas ou mais categorias ao mesmo tempo, o que prejudica o critério de exclusividade da classificação proposta. (b) A dimensão Quadro de Referência, não esclarece suficientemente o modo de identificação do quadro de referência utilizado, tomando-se como base a descrição metodológica da pesquisa. No caso de fontes de dados primárias e subjetivas de satisfação, por exemplo, não é possível identificar o quadro de referência do respondente, a menos que o tipo de pergunta aplicada na pesquisa esteja suficientemente descrita por seus autores. (c) A dimensão Unidade de Análise não é suficientemente exaustiva quanto às categorias propostas. (d) A dimensão Estrutura dos Indicadores apresenta limitação de aplicação prática, na medida em que a 
aplicação da classificação proposta depende da informação, nem sempre disponível, a respeito da direção de causalidade considerada pelos autores das pesquisas.

A despeito de suas limitações, o esquema conceitual de Carneiro et al. (2005) contribui para o avanço da discussão a respeito da multidimensionalidade do construto desempenho organizacional e seu status nos campos de pesquisa relacionados - direta ou indiretamente - às áreas de estudos de organizações e de estratégia. Contribui também para esclarecer dificuldades práticas e metodológicas do acesso multidimensional ao conceito no contexto de pesquisas científicas, chamando a atenção para a necessidade de reconhecimento dos indicadores adequados aos pressupostos teóricos das pesquisas empíricas. Dessa forma, defendem os autores, garante-se a validade das pesquisas e a possibilidade de comparação entre resultados obtidos por diferentes investigações.

Mais recentemente, Richard et al. (2009) analisaram o uso do conceito desempenho em 213 artigos publicados na Academy of Management Journal, Administrative Science Quarterly, Journal of International Business Studies, Journal of Management e Strategic Management Journal. Os autores realizaram a análise com base em sistema de classificação multidimensional composto pelas seguintes categorias: (a) uso do desempenho como variável dependente, independente ou de controle; (b) fonte dos dados (primários, secundários ou ambos); (c) operacionalização (objetivo, subjetivo, quaseobjetivo); (d) tipo de indicador (contábil, financeiro, reputação, vendas etc.); (e) quantidade de medidas (única, múltiplas); (f) tipo de análise (múltipla em separado; agregada por análise fatorial; agregada por média); e (g) corte temporal (longitudinal ou transversal).

Em todos os modelos de classificação propostos observam-se problemas relacionados à exclusividade e à exaustividade das categorias propostas. Por exemplo, os modelos analisados não incluem categorias de análise da abordagem teórica utilizada ou dos padrões de interação causal do desempenho com as demais variáveis da pesquisa. Além disso, diversas categorias propostas pelos modelos de classificação analisados não são exaustivas, por considerarem apenas as ocorrências que foram verificadas nas pesquisas e desconsiderarem outros desdobramentos teóricos possíveis.

\section{Descrição do Modelo Multidimensional de Análise do Conceito Desempenho}

O Modelo Multidimensional de Análise do Conceito Desempenho constitui-se em um esquema de classificação desenvolvido a partir de análise e síntese de atributos e propriedades do conceito de desempenho, inferidos a partir de análise de conteúdo de trabalhos anteriores e de revisão de literatura das áreas de organizações e de estratégia. Entre os trabalhos que serviram de base teórica e metodológica para o desenvolvimento do esquema de classificação apresentado a seguir, destaquem-se Cameron e Whetten (1983), Baron e Kenny (1986), Bailey (1994), Glunk e Wilderom (1999), Katsikeas et al. (2000), Klein e Kozlowski (2000), Vasconcelos (2002), Creswell (2002), Carneiro et al. (2005), Carneiro, Silva e Rocha (2007) e Richard et al. (2009).

Os atributos foram identificados e agrupados em propriedades, agrupadas de acordo com suas similaridades e diferenças, dando origem a três dimensões: Aspectos Conceituais (significados atribuídos ao conceito); Aspectos Formais (operacionalização do conceito em termos morfológicos); e Aspectos Relacionais (operacionalização do conceito em termos relacionais). As propriedades, portanto, correspondem a conjuntos de atributos diretamente observáveis do construto, enquanto as dimensões correspondem ao resultado da agregação das propriedades em conjuntos mais amplos, de acordo com similaridades e diferenças entre suas características.

Para refinamento do esquema de classificação multidimensional, uma primeira versão do Modelo Multidimensional de Análise do Conceito Desempenho foi submetida a pré-teste por meio do método de comparação constante, a partir da análise de 169 pesquisas empíricas das áreas de organizações de estratégia, selecionadas a partir das publicações RAE, RAC, RAUSP, Anais do ENANPAD 2005 a 2007, Strategic Management Journal, Organization Science, Administrative 
Science Quarterly e The Academy of Management Journal. O critério de seleção foi a utilização do conceito desempenho no desenho da pesquisa; o período analisado abrange os anos de 2005 a 2007. O método utilizado é similar ao utilizado pela ground theory, no qual as categorias de análise e agregação dos dados surgem a partir de comparação interna - entre similaridades e diferenças - e conforme o grau de conhecimento científico na área de estudo. Em áreas em que o estado do conhecimento é mais avançado, é possível encontrar categorias theory driven, ou seja, a partir de conceitos e relações entre conceitos anteriormente estabelecidos. O modelo de análise descrito a seguir, portanto, utiliza elementos e relações já estabelecidas anteriormente (theory driven) e propõe novos elementos e relações com base em levantamento bibliográfico e análise de conteúdo de pesquisas empíricas selecionadas para realização de pré-teste (ground theory).

A Figura 1 ilustra o Modelo Multidimensional de Análise do Conceito Desempenho.

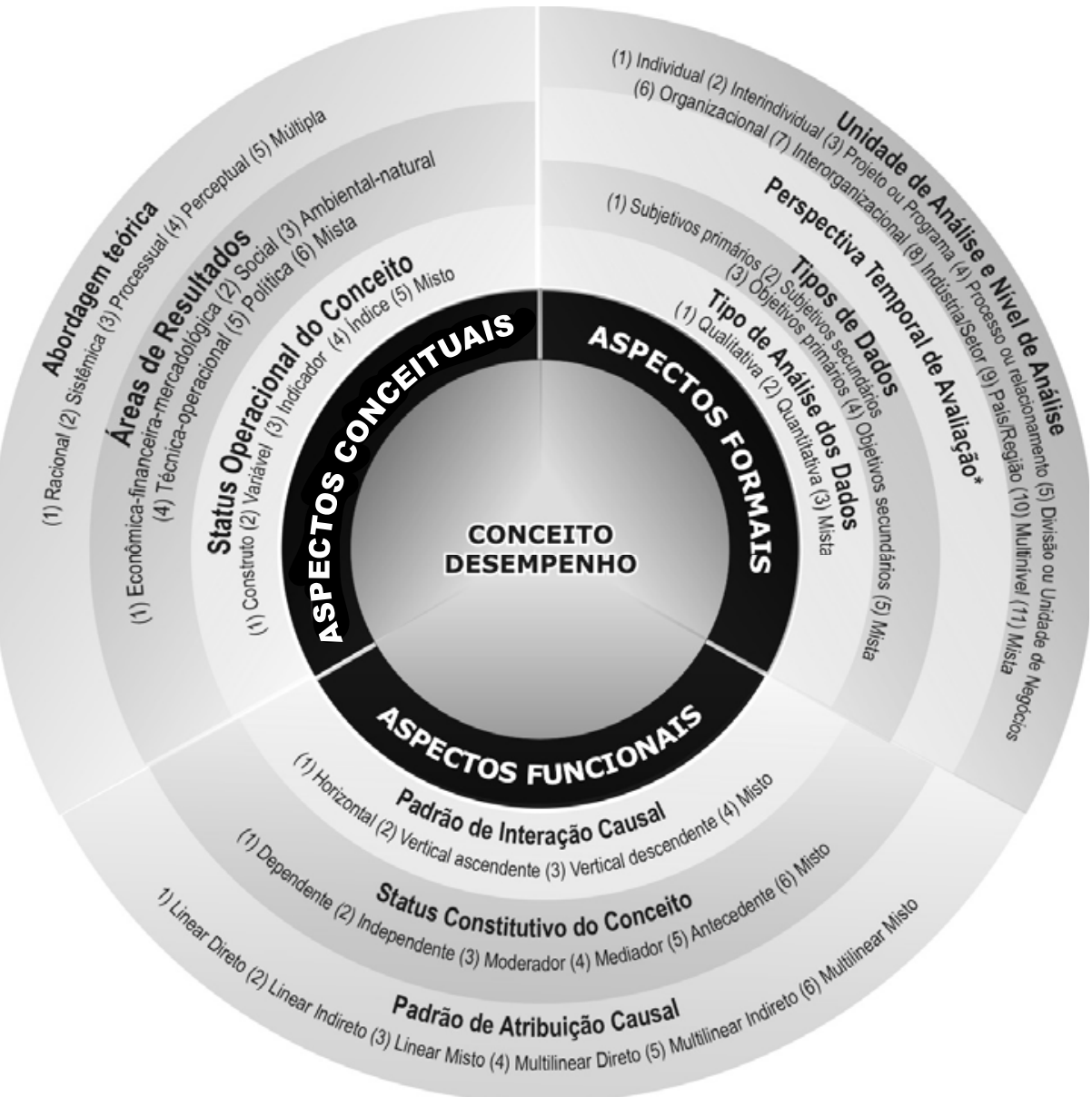

Figura 1. Modelo Multidimensional de Análise do Conceito Desempenho.

Nota. *(1) Estática cumulativa não-comparativa prospectiva; (2) Estática cumulativa não-comparativa retrospectiva; (3) Estática não-cumulativa comparativa prospectiva; (4) Estática não-cumulativa comparativa retrospectiva; (5) Estática nãocumulativa não-comparativa prospectiva; (6) Estática não-cumulativa não-comparativa retrospectiva; (7) Estática nãocumulativa não-comparativa atual; (8) Estática cumulativa comparativa prospectiva; (9) Estática cumulativa comparativa retrospectiva; (10) Dinâmica cumulativa não-comparativa prospectiva; (11) Dinâmica cumulativa não-comparativa retrospectiva; (12) Dinâmica não-cumulativa comparativa prospectiva; (13) Dinâmica não-cumulativa comparativa retrospectiva; (14) Dinâmica não-cumulativa não-comparativa perspectiva; (15) Dinâmica não-cumulativa não-comparativa retrospectiva; (16) Dinâmica não-cumulativa não-comparativa atual; (17) Dinâmica cumulativa comparativa perspectiva; (18) Dinâmica cumulativa comparativa retrospectiva; (19) Mista: utilização de múltiplas perspectivas temporais.

A seguir, estão descritas as propriedades e atributos correspondentes às dimensões propostas.

Status operacional do conceito. Natureza do conceito desempenho de acordo com a definição operacional. Atributos: (a) Construto corresponde à definição abstrata do conceito, obrigatoriamente operacionalizada por meio da agregação de variáveis e, opcionalmente, por meio da inclusão de 
indicadores e/ou índices. (b) Variável corresponde à definição abstrata do conceito e operacionalizada por meio da agregação de indicadores e/ou índices. (c) Indicador corresponde à definição empírica do conceito, mensurável e não agregada. (d) Índice corresponde à definição empírica do conceito, mensurável e formada por elementos agregados. (e) Misto.

Áreas de resultado. Natureza do conceito de desempenho de acordo com áreas potenciais de impacto e/ou avaliação das ações organizacionais, divididas conforme o foco predominante da definição operacional utilizada na pesquisa. Atributos: (a) Econômico-financeiro-mercadológica: avaliação do desempenho com base nos resultados da aplicação dos recursos de produção em termos de geração de valor econômico, financeiro e/ou mercadológico. (b) Social: avaliação do resultado com base em mudanças provocadas em indivíduos ou grupos sociais. (c) Ambiental-natural: avaliação do desempenho com base no uso e/ou conservação de recursos naturais. (d) Técnico-operacional: avaliação do desempenho com base em processos ou atributos internos. (e) Política: avaliação do resultado com base no poder de influência. (f) Mista: utilização simultânea de duas ou mais áreas de resultado organizacional.

Abordagem teórica. Perspectiva teórica, que serve como base para a definição operacional do conceito de desempenho. Atributos: (a) Racional com foco em objetivos, quando enfatiza as saídas (outputs) e o grau de concretização de objetivos predeterminados. (b) Sistêmica com foco no acesso a recursos, quando enfatiza as entradas (inputs) em lugar das saídas (outputs) e busca verificar a capacidade de acesso e uso de recursos. (c) Processual com foco nos processos internos, quando busca mensurar o desempenho com base em propriedades e/ou características organizacionais decorrentes e/ou provenientes do processo de conversão das entradas (inputs) em saídas (outputs). (d) Perceptual, quando busca mensurar o grau de satisfação de um ou mais stakeholders em relação ao desempenho. (e) Múltipla, quando são utilizados simultaneamente critérios de duas ou mais perspectivas teóricas.

Tipos de dados. Natureza dos dados utilizados para mensuração do desempenho, quanto ao grau de dependência em relação à fonte dos dados e ao grau de originalidade em relação à disponibilidade dos dados. Atributos: (a) Subjetivos primários, quando denotam opinião e/ou percepção individual e/ou agregada e são inéditos. (b) Subjetivos secundários, quando denotam opinião e/ou percepção individual e/ou agregada e não são inéditos. (c) Objetivos primários, quando os dados são independentes de opinião e/ou percepção individual e/ou agregada e são inéditos. (d) Objetivos secundários, quando os dados são independentes de opinião e/ou percepção individual e/ou agregada e não são inéditos. (e) Mistos, quando há presença simultânea de dados objetivos e subjetivos e/ou primários e secundários.

Unidade de análise. Nível de observação utilizado para coleta dos dados, quanto ao grau de interdependência dos seus elementos. Atributos: (a) Individual, quando a origem dos dados são indivíduos considerados independentes dos si em relação ao nível de análise da pesquisa. (b) Interindividual, quando a origem dos dados são indivíduos considerados interdependentes entre si em relação ao nível de análise da pesquisa. (c) Projeto ou programa, quando a origem dos dados é um subconjunto de ações organizacionais e espaciotemporalmente delimitadas, e cujas metas/objetivos são previamente conhecidas. (d) Processo ou relacionamento, quando a origem dos dados é um subconjunto de ações organizacionais espaciotemporalmente delimitadas, e cujas metas/objetivos não são previamente conhecidas. (e) Divisão ou unidade de negócios, quando a origem dos dados é uma área organizacional não delimitada temporalmente e hierarquicamente subordinada à direção central da organização. (f) Organizacional, quando a origem dos dados é o conjunto das áreas organizacionais não delimitadas temporalmente, cujas metas/objetivos são pretendidas e/ou não pretendidas. (g) Interorganizacional, quando a origem dos dados é formada por duas ou mais organizações independentes entre si, ou quando a origem dos dados é o conjunto das interações de duas ou mais organizações. (h) Indústria/setor, quando a origem dos dados é conjunto delimitado de organizações, definido a partir semelhanças entre objetivos e/ou área de atuação. (i) País/região, quando a origem dos dados é um conjunto geograficamente e politicamente delimitado de organizações. (j) Multinível, quando a origem dos dados é formada por duas ou mais unidades de análise. 
Nível de análise. Nível de generalização ou inferência utilizado para a mensuração do conceito, quanto ao grau de interdependência dos seus elementos. Atributos: (a) Individual, quando se pretende inferir resultados agregados obtidos por indivíduos considerados independentes entre si. (b) Interindividual, quando se pretende inferir resultados agregados obtidos por indivíduos considerados interdependentes. (c) Projeto ou programa, quando se pretende inferir resultados obtidos por um subconjunto de ações organizacionais espaciotemporalmente delimitadas, e cujas metas/objetivos são previamente conhecidas. (d) Processo ou relacionamento, quando se pretende inferir resultados obtidos por um subconjunto de ações organizacionais espaciotemporalmente delimitadas, e cujas metas/objetivos não são previamente conhecidas. (e) Divisão ou unidade de negócios, quando se pretende inferir resultados obtidos por uma área organizacional não delimitada temporalmente e hierarquicamente subordinada à direção central da organização. (f) Organizacional, quando se pretende inferir resultados obtidos pelo conjunto das áreas organizações não delimitadas temporalmente, cujas metas/objetivos são pretendidas e/ou não pretendidas. (g) Interorganizacional, quando se pretende inferir resultados obtidos por duas ou mais organizações independentes entre si, ou quando se pretende inferir resultados obtidos a partir da interação de duas ou mais organizações. (h) Indústria/setor, quando se pretende inferir resultados obtidos por um conjunto delimitado de organizações, definido a partir semelhanças entre objetivos e/ou área de atuação. (i) País/região, quando se pretende inferir resultados obtidos por um conjunto geograficamente e politicamente delimitado de organizações. (j) Multinível, quando se pretende inferir resultados agregados obtidos por dois ou mais níveis de análise. (k) Misto, quando se pretende inferir resultados desagregados obtidos por dois ou mais níveis de análise.

Tipo de análise dos dados. Resultado agregado das escolhas do pesquisador em relação à natureza da realidade, ao papel da ciência na apreensão dos fenômenos da realidade, aos critérios de legitimidade na escolha dos problemas e soluções a serem buscados no campo científico e aos critérios para a escolha dos métodos e técnicas de pesquisa mais adequadas a cada caso. Atributos. (a) Qualitativa: diz respeito à busca pela compreensão abrangente a respeito de um determinado fenômeno, a partir de múltiplas perspectivas e com base em processo predominantemente indutivo de construção de cenários, levantamento de problemas e construção de hipóteses de pesquisa. (b) Quantitativa: diz respeito à busca pela verificação empírica de hipóteses formuladas a partir de problemas já identificados no contexto de uma teoria, com o propósito de desenvolver explicações generalizáveis, a partir do uso de técnicas de mensuração e análise estatística. (c) Multimétodo: diz respeito à análise baseada ao mesmo tempo em pressupostos e inferências de base qualitativa e quantitativa.

Perspectiva temporal. Corte temporal utilizado na mensuração do conceito. Atributos. (a) Estática cumulativa não-comparativa prospectiva: mensuração atual cumulativa de medidas retrospectivas com o objetivo de inferir o resultado potencial futuro. (b) Estática cumulativa nãocomparativa retrospectiva: mensuração atual cumulativa de medidas retrospectivas com o objetivo de inferir o resultado passado. (c) Estática não-cumulativa comparativa prospectiva: mensuração atual não-cumulativa comparativa de medidas retrospectivas com o objetivo de inferir o resultado potencial futuro. (d) Estática não-cumulativa comparativa retrospectiva: mensuração atual não-cumulativa comparativa de medidas retrospectivas com o objetivo de inferir o resultado passado. (e) Estática nãocumulativa não-comparativa prospectiva: mensuração atual não-cumulativa e não-comparativa de medidas atuais com o objetivo de inferir o resultado potencial futuro. (f) Estática não-cumulativa nãocomparativa retrospectiva: mensuração atual não-cumulativa e não-comparativa de medidas atuais com o objetivo de inferir o resultado passado. (g) Estática não-cumulativa não-comparativa atual: mensuração atual não-cumulativa e não-comparativa de medidas atuais. (h) Estática cumulativa comparativa prospectiva: mensuração atual cumulativa e comparativa de medidas retrospectivas com 0 objetivo de inferir o resultado potencial futuro. (i) Estática cumulativa comparativa retrospectiva: mensuração atual cumulativa e comparativa de medidas de medidas retrospectivas com o objetivo de inferir o resultado passado. (j) Dinâmica cumulativa não-comparativa prospectiva: mensuração atual cumulativa da evolução de medidas retrospectivas com o objetivo de inferir o resultado potencial futuro. (k) Dinâmica cumulativa não-comparativa retrospectiva: mensuração atual cumulativa da evolução de medidas retrospectivas com o objetivo de inferir o resultado passado. (l) Dinâmica não- 
cumulativa comparativa prospectiva: mensuração atual não-cumulativa e comparativa da evolução de medidas retrospectivas com o objetivo de inferir o resultado potencial futuro. (m) Dinâmica nãocumulativa comparativa retrospectiva: mensuração atual não-cumulativa e comparativa da evolução de medidas retrospectivas com o objetivo de inferir o resultado passado. (n) Dinâmica não-cumulativa não-comparativa prospectiva: mensuração atual não-cumulativa e não-comparativa da evolução de medidas retrospectivas com o objetivo de inferir o resultado potencial futuro. (o) Dinâmica nãocumulativa não-comparativa retrospectiva: mensuração atual não-cumulativa e não-comparativa da evolução de medidas retrospectivas com o objetivo de inferir o resultado passado. (p) Dinâmica nãocumulativa não-comparativa atual: mensuração atual não-cumulativa e não-comparativa da evolução de medidas atuais. (q) Dinâmica cumulativa comparativa prospectiva: mensuração atual cumulativa comparativa da evolução de medidas retrospectivas com o objetivo de inferir o resultado potencial futuro. (r) Dinâmica cumulativa comparativa retrospectiva: mensuração atual cumulativa comparativa da evolução de medidas retrospectivas com o objetivo de inferir o resultado passado. (s) Mista: utilização de múltiplas perspectivas temporais.

Padrão de atribuição causal. (a) Quantidade de relações de causalidade estabelecidas; (b) grau de interdependência das relações de causalidade estabelecidas; e (c) presença de conceitos intervenientes na composição das relações de causalidade estabelecidas. Atributos. (a) Linear direto, quando as relações de causalidade entre o desempenho e as outras variáveis ou categorias de análise da pesquisa são únicas, ou seja, presume-se uma única causa e um único efeito e não há presença de variáveis mediadoras e/ou moderadoras em nenhuma das relações observadas. (b) Linear indireto, quando as relações de causalidade entre o desempenho e as outras variáveis ou categorias de análise da pesquisa são únicas e há presença de variáveis mediadoras e/ou moderadoras. (c) Linear misto, quando as relações de causalidade entre o desempenho e as outras variáveis ou categorias de análise da pesquisa são múltiplas, interdependentes e há presença de variáveis mediadoras e/ou moderadoras em pelo menos uma das relações observadas. (d) Multilinear direto, quando as relações de causalidade entre o desempenho e as outras variáveis ou categorias de análise da pesquisa são múltiplas, independentes entre si e não há presença de variáveis mediadoras e/ou moderadoras em nenhuma das relações observadas. (e) Multilinear indireto, quando as relações de causalidade entre o desempenho e as outras variáveis ou categorias de análise da pesquisa são múltiplas, independentes entre si e há presença de variáveis mediadoras e/ou moderadoras em todas as relações observadas. (f) Multilinear misto, quando as relações de causalidade entre o desempenho e as outras variáveis ou categorias de análise da pesquisa são múltiplas, independentes entre si e há presença de variáveis mediadoras e/ou moderadoras em pelo menos uma das relações observadas.

Status constitutivo do conceito. Função do conceito de desempenho no desenho da pesquisa, em relação à direção das relações de causalidade estabelecidas entre os conceitos. Atributos. (a) Dependente: quando o desempenho é considerado o efeito no contexto das relações de causalidade estabelecidas. (b) Independente: quando o desempenho é considerado a causa no contexto das relações de causalidade estabelecidas. (c) Moderador: quando se considera que o desempenho interage com os demais conceitos observados na pesquisa, intensificando ou enfraquecendo o resultado da interação. (d) Mediador: quando se considera que o desempenho é ao mesmo tempo o efeito de uma variável antecedente (independente) e a causa da variável dependente. (e) Antecedente: quando se considera que o desempenho antecede a ocorrência de outros fenômenos observados. (f) Misto: quando o desempenho assume duas ou mais funções simultâneas no desenho da pesquisa.

Padrão de interação causal. Tipos de hierarquia entre os níveis de análise utilizados na pesquisa. Atributos. (a) Horizontal: quando o nível de análise dos conceitos com os quais são estabelecidas relações de causalidade são idênticos aos níveis de análise a partir dos quais foram realizadas inferências a respeito do conceito de desempenho. (b) Vertical ascendente: quando o nível de análise dos conceitos com os quais são estabelecidas relações de causalidade são inferiores aos níveis de análise a partir dos quais foram realizadas inferências a respeito do conceito de desempenho, em relação ao seu grau de agregação. (c) Vertical descendente: quando o nível de análise dos conceitos com os quais são estabelecidas relações de causalidade são superiores aos níveis de análise a partir dos quais foram realizadas inferências a respeito do conceito de desempenho, em relação ao seu grau de 
agregação. (d) Misto: quando são utilizados níveis de análise inferiores e superiores ao nível de análise a partir dos quais foram realizadas inferências a respeito do conceito de desempenho, em relação ao seu grau de agregação.

\section{Análise Comparativa de Pesquisas Empíricas Brasileiras e Estrangeiras com Base no Modelo Multidimensional de Análise do Conceito Desempenho}

O objetivo desta etapa do trabalho, conforme apontado anteriormente, constitui-se na comparação de pesquisas empíricas brasileiras e estrangeiras das áreas de estudos de organizações e de estratégia, com base na descrição dos atributos, propriedades e dimensões propostos no Modelo Multidimensional de Análise do Conceito Desempenho. Busca-se, portanto, descrever as pesquisas com base (a) na frequência individual de utilização dos atributos e (b) na identificação de semelhanças e diferenças entre os grupos de pesquisa. A unidade de análise é constituída de material textual, classificado como comunicação formal, no sentido de que "a competência para sua realização exige um conhecimento especializado” (Bauer, Gaskell, \& Allum, 2005, p. 21). Quanto à técnica para coleta e tratamento dos dados, a presente pesquisa é classificada como análise de conteúdo categorial transnacional longitudinal. Quanto aos seus objetivos e métodos, a investigação é ao mesmo tempo descritivo-quantitativa e inferencial-qualitativa. De acordo com Bauer (2005, p. 190), "a análise de texto faz uma ponte entre um formalismo estatístico e a análise qualitativa dos materiais”. Trata-se, portanto, de uma técnica híbrida, na medida em que incorpora características das lógicas qualitativa e quantitativa.

O material textual utilizado para a análise de conteúdo foi selecionado de acordo com os critérios de construção de corpus, análogos àqueles aplicados na área da linguística. Morfologicamente, um corpus deve apresentar variação externa e variação interna. Enquanto a variação externa corresponde à capacidade de representar diferentes contextos ou fontes de consulta, a variação interna corresponde à capacidade de representar a diversidade temática, formal, funcional, de gênero, entre outras, que ela pretende representar. Neste trabalho, foram escolhidos trabalhos de pesquisa brasileiros e estrangeiros das áreas de estudos de organizações e de estratégia publicados durante o período de 2005 a 2007, os quais tenham utilizado o conceito desempenho no desenho da pesquisa, e nos quais tenham sido estabelecidas relações de causalidade, correlação ou associação entre os conceitos utilizados na investigação. De acordo com Bauer et al. (2005), os princípios básicos para o delineamento do corpus na seleção qualitativa são estes: relevância, homogeneidade e sincronicidade. A Tabela 1 apresenta a estrutura geral do corpus para análise de conteúdo, proposto para esta pesquisa.

Tabela 1

Estrutura Geral do Corpus para a Análise de Conteúdo

\begin{tabular}{ll}
\hline PUBLICAÇÃO & \multicolumn{1}{c}{ AUTORIA E ANO DE PUBLICAÇÃO ${ }^{(1,2,3)}$} \\
\hline RAE & Bertucci (2005); Fernandes, Fleury e Mills (2006); Goldszmidt, Brito e Vasconcelos (2007a); \\
& Mello e Marcon (2006); Perin, Sampaio e Hooley (2007); Silva, Motta e Costa (2007) \\
RAC & Bandeira-de-Mello e Marcon (2005); Brito e Vasconcelos (2005a); Brito e Vasconcelos (2005b); \\
& Castro e Figueiredo (2005); Claro, Claro e Zylbersztajn (2005); Gonçalves e Quintella (2006) \\
RAUSP & Campos (2006); Klotzle e Thomé (2006); Miele e Zylbersztajn (2005) \\
Anais do & Brito e Brito (2005) Brito, Campos, Brito e Thomaz (2005); Diniz Pereira (2005); \\
ENANPAD 2005 & Fensterseifer e Wilk (2005); Gonçalves e Quintella (2005); Faria, Brandão e Silva (2005); \\
& Nasser-Carvalho (2005); Omaki (2005); Pilati e Borgesandrade (2005); Rogers, Mendes-da- \\
& Silva, Paula (2005); Salles e Dellagnelo (2005); Scheible e Bastos (2005); Souza Leão (2005) \\
\hline
\end{tabular}




\title{
Tabela 1 (continuação)
}

\begin{tabular}{|c|c|}
\hline PUBLICAÇÃO & AUTORIA E ANO DE PUBLICAÇÃOO ${ }^{(1,2,3)}$ \\
\hline Anais do & Brito (2006); Castanhar, Dias e Esperança (2006); Correa e Guimarães (2006); Dias (2006); \\
\hline ENANPA & $\begin{array}{l}\text { Fischer (2006); Fontanelle, Hoeltgebaum e Silveira (2006); Grzebieluckas, Alberton, Marcon } \\
\text { e Kruger (2006); Moraes (2006); Schmidt (2006); Soares, Abreu e Sampaio (2006); Souto e } \\
\text { Rego (2006); Souza e Cordeiro (2006); Spers e Wright (2006) }\end{array}$ \\
\hline
\end{tabular}

Anais do Bedani (2007); Dias e Gonçalves (2007); Garrido (2007); Goldszmidt, Brito e Vasconcelos ENANPAD 2007 (2007b); Gonçalves, Gosling e Lanna (2007); Grzebieluckas, Marcon e Alberton (2007); Russo e Macedo-Soares (2007); Scheible, Batos e Rodrigues (2007); Veit e Gonçalves (2007)

Strategic $\quad$ Barnett e Salomon (2006); Chacar e Vissa (2005); Chakrabarti, Singh e Mahmood (2007); Management Coombs e Gilley (2005); Crossland e Hambrick (2007); David, Bloom e Hillman (2007); Journal Desarbo, Di Benedetto, Song e Sinha (2005); Douma, George e Kabir (2006); Ebben e Johnson (2005); Echols e Tsai (2005); Eisenmann (2006); Fang, Wade, Delios e Beamish (2007); Goerzen e Beamish (2005); Gong, Shenkar, Luo e Nyaw (2007); Goold, Young e Collis (2007); Haas e Hansen (2005); Hayward e Shimizu (2006); Henderson, Miller e Hambrick (2006); Hough (2006); Hult, Ketchen e Arrfelt (2007); Karaevli (2007); Katsikeas, Samiee e Theodosiou (2006); Kor e Leblebici (2005); Kor e Mahoney (2005); Laursen e Salter (2006); Lavie (2007); Lazzarini (2007); Li e Zhang (2007); Love e Nohria (2005); Macher e Boerner (2006); Makri, Lane e Gomez-Mejia (2006); McNamara, Aime e Vaaler (2005); Moran (2005); Pehrsson (2006); Rothaermel, Hitt e Jobe (2006); Short, Ketchen, Palmer e Hult (2007); Sorenson, McEvily, Ren e Roy (2006); Tanriverdi e Venkatraman (2005); Zaheer e Bell (2005)

Organization Aral e Weill (2007); Bercovitz, Jap e Nickerson (2006); Cattani (2005); Espinosa, Kraut, Science $\quad$ Slaughter, Lerch e Herbsleb (2007); Greenwood, Li, Prakash e Deephouse (2005); Harris e Bromiley (2007); Leana e Pil (2006); Mayer e Nickerson (2005); Mehra, Dixon, Brass e Robertson (2006); Park (2007); Payne (2006); Quigley, Tesluk, Locke e Bartol (2007); Rothaermel e Hill (2005); Siegel e Hambrick (2005); Vera e Crossan (2005); Voss, Cable e Voss (2006); Yakubovich e Lup (2006)

Administrative Baum, Rowley, Shipilov e Chuang (2005); Chatterjee e Hambrick (2007); Côté e Miners Science (2006); Gulati e Sytch (2007); Luo e Chung (2005); Maurer e Ebers (2006); Westphal e Quarterly Bednar (2005)

The Academy of Agle, Nagarajan, Sonnenfeld e Srinivasan (2006); Bommer, Dierdorff e Rubin (2007); Management Bonardi, Holburn e Vanden Berg (2006); Boone, van Olffen e van Witteloostuijn (2005); Journal Carson, Tesluk e Marrone (2007); Collins e Smith (2006); Ellis (2006); George (2005); Haleblian, Kim e Rajagopalan (2006); Hoang e Rothaermel (2005); Hunter e Thatcher (2007); Joshi, Liao e Jackson (2006); Kacmar, Andrews, Rooy e Steilberg (2006); Kassinis e Vafeas (2006); Krishnan, Martin e Noordhaven (2006); Kroll, Walters e Le (2007); Labianca, Moon e Watt (2005); Lavie, Lechner e Harbir (2007); Mathieu e Schulze (2006); Mayer e Gavin (2005); Miller e Eden (2006); Porath e Erez (2007); Raver e Gelfand (2005); Russo e Harrison (2005); Sampson (2007); Sanders e Hambrick (2007); Shaw, Duffy, Johnson e Lockhart (2005); Shaw, Gupta e Delery (2005); Shipilov (2006); Sine, Mitsuhashi e Kirsch (2006); Srivastava, Bartol e Locke (2006); Sun, Aryee e Law (2007); Van der Vegt e Bunderson (2005); Von Nordenflycht (2007); Wade, Porac, Pollock e Graffin (2006); Wang, Law, Hackett, Wang e Chen (2005); Zellmer-Bruhn e Gibson (2006)

\begin{abstract}
Nota. (1) Do total de artigos estrangeiros selecionados inicialmente para compor o corpus da análise de conteúdo, 10 não foram localizados em bases de dados e 2 não apresentavam a descrição explícita das hipóteses, o que impossibilitou a análise completa. (2) No caso de pesquisas realizadas em duas fases, considerou-se apenas a(s) fase(s) em que o conceito desempenho fazia parte do desenho da investigação. (3) Alguns artigos apresentaram dois ou mais desenhos de pesquisa, portanto foram analisadas 169 pesquisas.
\end{abstract}

Quanto à escolha das publicações, foram selecionadas por conveniência, tomando-se também como base sua importância histórica e atual para as áreas de estudos de organizações e de estratégia. Os anais de congresso foram incluídos no corpus de pesquisas brasileiras em razão da baixa frequência de uso do conceito de desempenho nas revistas selecionadas. Buscou-se, portanto, maior equilíbrio quantitativo entre pesquisas brasileiras e estrangeiras. 
Quanto às limitações da pesquisa, ressalte-se que a pesquisa qualitativa carece de maior formalidade em termos de repertório e critérios de qualidade equivalentes aos tradicionalmente utilizados em pesquisas quantitativas. Ainda quanto à análise dos dados, há a dificuldade em captar, principalmente em se tratando de argumentos expressos sob a forma de texto, diferenças de estilo, de contexto ou de intenção potencialmente intervenientes no conteúdo proposto pelos autores. Além disso, a descrição incompleta ou ambígua de pressupostos teóricos, delineamentos metodológicos e procedimentos de coleta e análise dos dados prejudicam a interpretação do material textual que compõe o corpus.

\section{Apresentação dos dados da análise dos aspectos conceituais}

Os dados da propriedade Status Operacional do Conceito foram analisados em quatro etapas. (a) Todos os níveis de Análise das Pesquisas Brasileiras. (b) Nível de Análise Organizacional das Pesquisas Brasileiras. (c) Todos os níveis de Análise das Pesquisas Estrangeiras. (d) Nível de Análise Organizacional das Pesquisas Estrangeiras. A análise de dados das demais propriedades segue este mesmo padrão de apresentação.

Nas pesquisas brasileiras predomina o uso de variáveis (46\%) e indicadores (45\%), respectivamente, revelando-se equilíbrio entre a busca de controle e profundidade na mensuração do desempenho e o uso de medidas diretamente observáveis e não agregadas. Comparando-se os resultados obtidos para todos os níveis de análise aos resultados obtidos com a seleção das pesquisas do nível de análise organizacional - as quais correspondem a 83,9\% do total de pesquisas brasileiras observa-se uma inversão na frequência de uso de indicadores e variáveis, com predomínio do uso de indicadores $(53,2 \%)$ em relação ao uso de outros tipos de operacionalização do conceito. Nas pesquisas estrangeiras, verifica-se preferência pelo uso de indicadores para pesquisas de todos os níveis de análise $(71,1 \%)$ e para pesquisas do nível de análise organizacional (80,5\%), as quais correspondem a $67,5 \%$ do total de pesquisas estrangeiras. Conclui-se, portanto, haver preferência da maior parte dos autores do corpus de pesquisas estrangeiras pela observação direta de indicadores de desempenho, em detrimento do desenvolvimento de escalas múltiplas agregadas.

Quanto à propriedade Áreas de Resultado, observando-se as pesquisas brasileiras e considerando-se todos os níveis de análise, observa-se maior frequência do foco nos resultados econômico-financeiro-mercadológicos (64,9\%), ou seja, prevalece uma identificação conceitual do desempenho com os resultados da aplicação dos recursos de produção, em termos de geração de valor. Considerando-se apenas as pesquisas do nível de análise organizacional, permanece o predomínio do foco nos resultados econômico-financeiro-mercadológicos (74,5\%), enquanto 25,5\% das pesquisas utilizaram atributos técnico-operacionais de desempenho organizacional. Verifica-se, portanto, uma tendência do uso de atributos de áreas de resultados não-múltiplas, indicativo de uma atribuição de significado unidimensional ao conceito desempenho.

Nas pesquisas estrangeiras, considerando-se todos os níveis de análise, observa-se uma distribuição de frequência semelhante àquela encontrada nas pesquisas brasileiras, embora haja aumento na frequência de pesquisas com foco na área técnico-operacional e o surgimento de pesquisas com foco nas áreas ambiental-natural $(1,8 \%)$ e política $(0,9 \%)$, ainda que com baixa representatividade em relação ao total. Observa-se, também, a predominância do foco em resultados da área econômicofinanceiro-mercadológica, com um total de $87 \%$ de pesquisas.

Para a propriedade Abordagem Teórica predomina a abordagem racional nas pesquisas brasileiras (58,9\%), com ênfase nas saídas (outputs) e no grau de concretização de objetivos predeterminados. Percebe-se, portanto, uma tendência à observação do resultado organizacional agregado e posterior às transformações geradas pelos processos organizacionais internos e pelas atividades de acesso a recursos, ao mesmo tempo que há pouca ênfase na satisfação dos stakeholders, como critério de conceituação e mensuração de desempenho. Nas pesquisas brasileiras com abordagem teórica mista, prevalece a combinação de aspectos racionais com aspectos processuais e perceptuais, e observa-se pouca representatividade da abordagem sistêmica. Entre as pesquisas 
brasileiras do nível de análise organizacional, os resultados de frequência são semelhantes àqueles obtidos para o total de pesquisas, com substituição das pesquisas de foco processual pelas pesquisas de foco racional $(66 \%)$ e misto $(29,8 \%)$.

Entre as pesquisas estrangeiras, observa-se o predomínio da perspectiva teórica racional (71,9\%), seguida pela processual $(16,7 \%)$ e com baixa representatividade de outras perspectivas. Para as pesquisas estrangeiras do nível de análise organizacional diminui a frequência do foco processual, enquanto as outras abordagens permanecem representadas em proporções semelhantes àquelas encontradas para o total de pesquisas.

\section{Apresentação dos dados da análise dos aspectos formais}

Quanto à propriedade Tipos de Dados, conclui-se que há preferência por dados objetivos secundários $(51,8 \%)$ no corpus de pesquisas brasileiras de todos os níveis de análise, seguidos por dados subjetivos primários (30,4\%), distribuição semelhante àquela encontrada na descrição dos dados das pesquisas brasileiras do nível de análise organizacional.

Para as pesquisas estrangeiras, considerando-se todos os níveis de análise, predominam os dados objetivos secundários como fonte principal de dados (58,8\%), com distribuição de frequência semelhante entre dados objetivos primários $(19,3 \%)$ e subjetivos primários $(16,7 \%)$. Para as pesquisas estrangeiras do nível de análise organizacional, observa-se aumento no uso de dados objetivos secundários (81,8\%), possivelmente em razão da disponibilidade de bancos de dados públicos e privados. Observa-se, ainda, diminuição no uso de dados subjetivos primários em relação ao total de pesquisas, demonstrando baixa frequência de uso de informações perceptuais, opinativas e/ou avaliativas individuais para a coleta de dados de desempenho organizacional.

Para as propriedades Unidade de Análise e Nível de Análise verificou-se no corpus de pesquisas brasileiras predominância $(82,1 \%)$ da coleta de dados no nível da organização. Em apenas um caso utilizou-se como origem dos dados para mensuração do desempenho organizacional unidades de análise e nível de análise de origem distinta: a organização e a unidade de negócios. Nesse caso, as duas unidades de análise foram utilizadas simultaneamente - embora de forma não agregada - para inferir o desempenho da organização.

Quanto ao total de pesquisas estrangeiras, para todos os níveis de análise, observa-se maior diversidade no uso de unidades e níveis de análise, com destaque para os níveis organizacional $(65,8 \%)$, individual $(12,3 \%)$ e interindividual $(11,4 \%)$. Para pesquisas estrangeiras do nível de análise organizacional, observou-se apenas $2,6 \%$ de pesquisas que utilizaram dados do nível de análise projeto/programa e 1,3\% do nível de análise individual para realização de inferências a respeito do nível de análise organizacional. Conclui-se, portanto, que há predominância da mensuração do conceito desempenho em um único nível de análise, a partir de uma única unidade de análise.

Para a propriedade Tipo de Análise dos Dados predomina em ambos os grupos de pesquisas brasileiras em todos os níveis de análise, brasileiras no nível da análise organizacional, estrangeiras em todos os níveis de análise, estrangeiras do nível de análise organizacional - o uso dos métodos quantitativos: $92,9 \%, 91,5 \%, 99,1 \%$ e $98,7 \%$ respectivamente.

Quanto à propriedade Perspectiva Temporal, para o grupo de pesquisas brasileiras, em todos os níveis de análise, foram identificados $38 \%$ de pesquisas da perspectiva temporal estática nãocumulativa comparativa retrospectiva; $34 \%$ da perspectiva temporal estática não-cumulativa nãocomparativa retrospectiva, enquanto $23 \%$ de pesquisas utilizaram múltiplas perspectivas temporais. Para o grupo de pesquisas brasileiras do nível de análise organizacional, foram encontrados $47 \%$ de pesquisas da perspectiva temporal estática não-cumulativa comparativa retrospectiva, $27 \%$ da perspectiva temporal estática não-cumulativa não-comparativa retrospectiva, enquanto $23 \%$ de pesquisas utilizaram múltiplas perspectivas temporais. Verifica-se, portanto, preferência pela realização de observação atual de medidas retrospectivas ou retrospectivas comparativas, em detrimento do uso de mensurações evolutivas comparativas ou de mensurações prospectivas. Conclui- 
se, portanto, pela preferência por medidas estáticas e já disponíveis indicativas do desempenho passado, em detrimento de medidas dinâmicas evolutivas e/ou prospectivas de desempenho. Observase, também, baixa variação na distribuição de frequência das pesquisas brasileiras de todos os níveis de análise em comparação da distribuição de frequência das pesquisas de nível de análise organizacional.

Para o grupo de pesquisas estrangeiras, em todos os níveis de análise, foram identificados 38\% de pesquisas da perspectiva temporal estática não-cumulativa comparativa retrospectiva, $34 \%$ da perspectiva temporal estática não-cumulativa não-comparativa retrospectiva, $9 \%$ de pesquisas mistas a partir de múltiplas perspectivas temporais, $6 \%$ da perspectiva estática não-cumulativa não-comparativa retrospectiva, 5\% da perspectiva estática não-cumulativa não-comparativa atual, 2,5\% da perspectiva dinâmica não-cumulativa comparativa retrospectiva e 2,5\% da perspectiva estática não-cumulativa comparativa prospectiva. Para o grupo de pesquisas estrangeiras do nível de análise organizacional, foram identificados $47 \%$ de pesquisas da perspectiva temporal estática não-cumulativa comparativa retrospectiva, $21 \%$ da perspectiva temporal estática não-cumulativa não-comparativa retrospectiva, $10 \%$ da perspectiva mista, $9 \%$ da perspectiva estática cumulativa não-comparativa retrospectiva, $4 \%$ da perspectiva não-cumulativa comparativa retrospectiva e $4 \%$ da perspectiva estática não-cumulativa comparativa prospectiva. Ambas as distribuições de frequência são similares àquelas encontradas para o grupo de pesquisas brasileiras, com exceção da existência de maior diversidade no uso das perspectivas temporais, a despeito da baixa frequência de utilização da maior parte dos recortes temporais identificados. De forma geral, portanto, há baixa frequência de uso de medidas evolutivas e prospectivas, com ênfase para mensurações pontuais de medidas retrospectivas cumulativas e/ou nãocumulativas.

\section{Apresentação dos dados da análise dos aspectos relacionais}

Para a propriedade Padrão de Atribuição Causal, nas pesquisas brasileiras em todos os níveis de análise, observa-se predominância de relações Multilineares Diretas (73,2\%), fator indicativo da presença de relações múltiplas, independentes entre si e sem uso de conceitos mediadores e/ou moderadores na maior parte das pesquisas observadas. Para o conjunto de pesquisas do nível de análise organizacional, as frequências observadas apresentam pouca alteração.

Para o grupo de pesquisas estrangeiras, identificou-se maior similaridade entre as frequências das relações multilineares indiretas nos dois conjuntos de pesquisas - 45,6\% e 36,4\% respectivamente - das multilineares diretas - 31,6\% e 35,1\% respectivamente - e das multilineares mistas, com $17,5 \%$ e 22,1\% respectivamente. A implicação teórica dos padrões de atribuição causal observados consiste na constatação de há um estabelecimento de relações causais mais complexas no grupo de pesquisas estrangeiras, conforme se observa pela maior frequência de uso de conceitos mediadores e moderadores. O predomínio de relações multilineares, para os dois conjuntos de pesquisas, também permite inferir o reconhecimento, por parte dos autores, da existência simultânea de múltiplas relações de causalidade entre o desempenho e os demais fatores observados nas pesquisas.

Na descrição da distribuição de frequência dos atributos para a propriedade Status Constitutivo do Conceito, observa-se uma característica já apontada em pesquisas anteriores, ou seja, a predominância do uso do conceito desempenho como efeito das relações de causalidade estabelecidas no campo de estudos organizacionais e de estratégia. Para as quatro amostras a frequência superou os $80 \%$ de ocorrência. Observa-se, ainda, maior diversidade no uso do conceito entre as pesquisas estrangeiras, onde aparece como moderador, mediador, antecedente, misto e independente.

A última propriedade observada, o Padrão de Interação Causal, permite observar o uso de múltiplos níveis nas inferências causais aplicadas no desenho da pesquisa. Nas pesquisas brasileiras predomina a horizontalidade (48\%) como padrão de interação causal, ou seja, quase metade das pesquisas considerou como causas ou efeitos do desempenho conceitos mensurados no mesmo nível de análise utilizado para a mensuração do conceito desempenho. 
Em segundo lugar, com 36\% de frequência, foram utilizados ao mesmo tempo conceitos do nível de mensuração de desempenho e conceitos de outros níveis de análise. Em um grupo menor de pesquisas, optou-se por utilizar apenas conceitos de nível de análise inferior (10,7\%) ou superior (5,4\%). Para o grupo de pesquisas brasileiras do nível de análise organizacional, as frequências encontradas são semelhantes, de onde se infere uma ênfase teórica nas explicações do desempenho organizacional, com base em fatores organizacionais. Em outras palavras, elementos e fenômenos relacionados à própria organização são considerados como determinantes do desempenho organizacional. Quanto às pesquisas estrangeiras, observa-se uma distribuição mais equilibrada entre as distribuições de frequência das interações causais dos diversos níveis de análise, principalmente quanto às pesquisas de orientação vertical. Conclui-se, portanto, que há maior variedade entre os níveis de análise utilizados como causa e efeito no grupo de pesquisas estrangeiras.

\section{Principais Contribuições do Modelo Multidimensional de Análise do Conceito Desempenho e Sugestões para Estudos Futuros}

Um aperfeiçoamento nos métodos de mensuração do conceito desempenho depende do aperfeiçoamento na compreensão das implicações teóricas e metodológicas da multidimensionalidade do construto (Richard, Devinney, Yip, \& Johnson, 2009). Como lembram os autores, "O desempenho organizacional é a variável dependente final de interesse de pesquisadores preocupados com praticamente qualquer área da administração" (p. 719). Entretanto a importância do desempenho organizacional não se traduz em uma preocupação generalizada com as escolhas teóricas e metodológicas envolvidas na conceituação e mensuração do conceito. O uso de indicadores únicos ou não agregados para mensuração do desempenho em pesquisas empíricas é prática comum e é um demonstrativo dessa fragilidade. E esse quadro se agrava ainda mais, se considerarmos o surgimento de novos indicadores de desempenho - tais como o desempenho social ou o desempenho ambiental particularmente nas últimas duas décadas. Ferramentas gerenciais tais como o Balanced Scorecard também chamam a atenção para a multidimensionalidade do desempenho organizacional.

O Modelo Multidimensional de Análise do Conceito Desempenho, aqui proposto, possibilita a visualização das possibilidades de conceituação e operacionalização do conceito na medida em que: (a) apresenta diferentes possibilidades de atribuição conceitual; (b) apresenta diferentes possibilidades de atribuição formal; e (c) apresenta diferentes possibilidades de atribuição relacional. Em comparação das propostas de classificação anteriores, o esquema aqui proposto também constitui um avanço: (a) ao incorporar contribuições das teorias de efetividade e desempenho organizacional, propostas pela literatura das áreas de organizações e de estratégia; (b) ao superar algumas das limitações dos esquemas de classificação de base empírica, principalmente em relação à exaustividade e exclusividade das categorias da análise e à fertilidade dos resultados obtidos; e (c) ao incluir categorias de análise, relacionadas aos padrões de atribuição e interação causal.

A partir da análise do instrumento e das características próprias de cada quadro teórico, cabe a cada pesquisador desenvolver as escolhas conceituais, formais e relacionais mais adequadas ao desenho da pesquisa. Concordamos, portanto, com o ponto de vista de Steers (1975), Cameron e Whetten (1983), os quais já chamaram a atenção para a necessidade de contextualização da conceituação e mensuração do conceito de desempenho. Além disso, um dos grandes desafios dos pesquisadores da área de organizações e de estratégia é o desenvolvimento de estratégias metodológicas que deem conta da multidimensionalidade do conceito desempenho. Nosso objetivo aqui, portanto, foi alcançado, ao demonstrarmos múltiplas dimensões que devem ser consideradas no delineamento das pesquisas.

O próximo passo é o desenvolvimento de novas pesquisas a respeito do modo como o conceito tem sido mensurado e também do modo como deve ser mensurado no futuro. Sugerimos, portanto, que futuros estudos demonstrem as vantagens e desvantagens de diferentes combinações dos atributos e propriedades aqui apresentados, como forma de refinamento de escalas alternativas de mensuração do 
conceito desempenho. Por exemplo, quais são as implicações teóricas e metodológicas do uso de índices ambientais-naturais objetivos secundários para mensuração do desempenho interorganizacional? Ou, ainda, existem combinações de propriedades atributos mais apropriadas para determinados tipos de organizações, indústrias ou ambientes? Existem diferenças entre as combinações de atributos, utilizadas pelos pesquisadores em pesquisas antigas e pesquisas recentes?

Sugerimos, também, que o modelo de análise multidimensional, aqui proposto, seja revisado e ampliado, de forma a incluir categorias de análise para a representação da dimensão epistemológica das pesquisas e para a representação da estrutura formativa ou refletiva dos indicadores de variáveis latentes.

Finalmente, acreditamos que compreender a natureza do conceito de desempenho contribui para a superação do desafio teórico contínuo da compreensão das complexidades organizacionais. E, para finalizar este trabalho, reproduzimos as palavras de Russo e Harrison (2005, p. 590), pesquisadores da University of Oregon e autores de um dos artigos do corpus de pesquisas estrangeiras aqui analisadas:

Em lugar de procurar por algum 'Santo Graal' explicativo, os pesquisadores deveriam encarar cada análise como uma peça de um quebra-cabeça. Cada peça tem valor limitado quando considerada isoladamente, mas demonstra sua utilidade quando as peças são colocadas em seus devidos lugares. Nosso objetivo neste estudo exploratório foi contribuir com mais uma peça de um quebra-cabeça que vai incomodar, surpreender e, quem sabe até, esperamos, inspirar acadêmicos na medida em que o quadro for se revelando.

Artigo recebido em 09.02.2010. Aprovado em 17.12.2010.

\section{Referências}

Agle, B. R., Nagarajan, N. J., Sonnenfeld, J. A., \& Srinivasan, D. (2006). Does CEO charisma matter? An empirical analysis of the relationships among organizational performance, environmental uncertainty, and top management teams' perceptions of CEO charisma. Academy of Management Journal, 49(1), 161-174.

Aral, S., \& Weill, P. (2007). IT Assets, organizational capabilities, and firm performance: how resource allocations and organizational differences explain performance variation. Organization Science, 18(5), 763-780. doi: 10.1287/orsc.1070.0306

Bailey, K. D. (1994). Typologies and taxonomies: an introduction to classification techniques (Série Quantitative Applications in the Social Sciences). Thousand Oaks, CA: Sage Publications.

Bandeira-de-Mello, R., \& Marcon, R. (2005). Unpacking firm effects: modeling political alliances in variance decomposition of firm performance in turbulent environments [Edição Especial]. Revista de Administração Contemporânea, 9, 41-64. doi: 10.1590/S1415-65552005000500003

Barnett, M. L., \& Salomon, R. M. (2006). Beyond dichotomy: the curvilinear relationship between social responsibility and financial performance. Strategic Management Journal, 27(11), 11011122. doi: $10.1002 / \mathrm{smj} .557$

Baron, R. M., \& Kenny, D. A. (1986). The moderator-mediator variable distinction in social psychological research: conceptual, strategic and statistical considerations. Journal of Personality and Social Psychology, 51(6), 1173-1182. doi: 10.1037/0022-3514.51.6.1173

Bauer, M. W. (2005). Análise de conteúdo clássica: uma revisão. In M. W. Bauer \& G. Gaskell (Eds.), Pesquisa qualitativa com texto, imagem e som: um manual prático (P. A. Guareschi, Trad.). (4a ed., pp. 189-217). Petrópolis: Vozes. (Obra original publicada em 2000) 
Bauer, M., Gaskell, G., \& Allum, N. (2005). Qualidade, quantidade e interesses do conhecimento: evitando confusões. In M. W. Bauer \& G. Gaskell (Eds.), Pesquisa qualitativa com texto, imagem e som: um manual prático (P. A. Guareschi, Trad.). (4a ed., pp. 17-36). Petrópolis: Vozes. (Obra original publicada em 2000)

Baum, J. A. C., Rowley, T. J., Shipilov, A. V., \& Chuang, Y. T. (2005). Dancing with strangers: aspiration performance and the search for underwriting syndicate partners. Administrative Science Quarterly, 50(4), 536-575. doi: 10.2189/asqu.50.4.536

Bedani, M. (2007, setembro). Efetividade das equipes de trabalho: clima organizacional como variável preditora do desempenho. Anais do Encontro Nacional da Associação Nacional de PósGraduação e Pesquisa em Administração, Rio de Janeiro, RJ, Brasil, 31.

Bercovitz, J., Jap, S. D., \& Nickerson, J. A. (2006). The antecedents and performance implications of cooperative exchange norms. Organization Science, 17(6), 724-740. doi: 10.1287/orsc. 1060.0213

Bertucci, J. L. O. (2005). Ambiente, estratégia e performance organizacional no setor industrial e de serviços. Revista de Administração de Empresas, 45(3), 10-24. doi: 10.1590/S003475902005000300002

Bommer, W. H., Dierdorff, E. C., \& Rubin, R. S. (2007). Does prevalence mitigate relevance? The moderating effect of group-level OCB on employee performance. Academy of Management Journal, 50(6), 1481-1494. doi: 10.2307/20159485

Bonardi, J., Holburn, G., \& Vanden Berg, R. G. (2006). Nonmarket strategy performance: evidence from U.S. electric utilities. Academy of Management Journal, 49(6), 1209-1228.

Boone, C., Olffen, W. van, \& Witteloostuijn, A. van (2005). Team locus-of-control composition, leadership structure, information acquisition, and financial performance: a business simulation study. Academy of Management Journal, 48(5), 889-909.

Brito, E. P. Z., \& Brito, L. A. L. (2005, setembro). Tamanho e desempenho financeiro. Anais do Encontro Nacional da Associação Nacional de Pós-Graduação e Pesquisa em Administração, Brasília, DF, Brasil, 29.

Brito, E. P. Z., Campos, L. A., Brito, L. A. L., \& Thomaz, J. C. (2005, setembro). Reputação corporativa e desempenho: uma análise empírica no setor bancário. Anais do Encontro Nacional da Associação Nacional de Pós-Graduação e Pesquisa em Administração, Brasília, DF, Brasil, 29.

Brito, L. A. L. (2006, setembro). A multi level analysis of firm performance - the effect of firm size. Anais do Encontro Nacional da Associação Nacional de Pós-Graduação e Pesquisa em Administração, Salvador, BA, Brasil, 30.

Brito, L. A. L., \& Vasconcelos, F. C. (2005a). A influência do país de origem no desempenho das empresas. Revista de Administração Contemporânea, 9(4), 97-118. doi: 10.1590/S141565552005000400006

Brito, L. A. L., \& Vasconcelos, F. C. (2005b). Desempenho das empresas brasileiras: efeitos ano, ramo de negócios e firma individual [Edição Especial]. Revista de Administração Contemporânea, 9, 65-85. doi: 10.1590/S1415-65552005000500004

Cameron, K. S., \& Whetten, D. A. (1983). Organizational effectiveness: a comparison of multiple models. San Diego: Academic Press.

Campos, T. L. C. (2006). Estrutura de propriedade e desempenho econômico; uma avaliação empírica para as empresas de capital aberto no Brasil. Revista de Administração da USP, 41(4), 369-380. 
Carneiro, J., Silva, J. F., \& Rocha, A. (2007). A critical analysis of measurement models of export performance. Brazilian Administration Review, 4(2), 1-19. doi: 10.1590/S180776922007000200002

Carneiro, J., Silva, J. F., Rocha, A., \& Hemais, C. (2005, dezembro). Conceptualisation and measurement of business performance: a multidimensional approach. Anais do International Meeting of the Iberoamerican Academy of Management, Lisboa, Portugal, 4.

Carson, J. B., Tesluk, P. E., \& Marrone, J. A. (2007). Shared leadership in teams: an investigation of antecedent conditions and performance. Academy of Management Journal, 50(5), 1217-1234.

Castanhar, J. C., Dias, J. F., \& Esperança, J. P. (2006, setembro). Orientação empreendedora, reconhecimento de oportunidades e desempenho em pequenas e médias empresas brasileiras: evidências de dois estudos de caso. Anais do Encontro Nacional da Associação Nacional de Pós-Graduação e Pesquisa em Administração, Salvador, BA, Brasil, 30.

Castro, E. C., \& Figueiredo, P. N. (2005, maio) Aprendizagem tecnológica compensa? Implicações da acumulação de competências tecnológicas para o aprimoramento de performance técnicoeconômica em uma unidade de aciaria no Brasil (1997-2001) [Edição Especial]. Revista de Administração Contemporânea, 9, 109-133. doi: 10.1590/S1415-65552005000500006

Cattani, G. (2005). Pre-adaptation, firm heterogeneity and technological performance: a study on the evolution of fiber optics, 1970-1995. Organization Science, 16(6), 563-580. doi: 10.1287/orsc.1050.0145

Chacar, A., \& Vissa, B. (2005). Are emerging economies less efficient? Performance persistence and the impact of business group affiliation. Strategic Management Journal, 26(10), 933-946. doi: $10.1002 / \mathrm{smj} .478$

Chakrabarti, A., Singh, K., \& Mahmood, I. (2007). Diversification and performance: evidence from East Asian firms. Strategic Management Journal, 28(2), 101-120. doi: 10.1002/smj.572

Chatterjee, A., \& Hambrick, D. C. (2007). It's all about me: narcissistic CEOs and their effects on company strategy and performance. Administrative Science Quarterly, 52(3), 351-386.

Claro, D. P., Claro, P. B. O., \& Zylbersztajn, D. (2005). Relationship marketing strategies: when buyer and supplier follow different strategies to achieve performance [Edição Especial]. Revista de Administração Contemporânea, 9, 35-60. doi: 10.1590/S1415-65552005000600003

Collins, C. J., \& Smith, K. G. (2006). Knowledge exchange and combination: the role of human resource practices in the performance of high-technology firms. Academy of Management Journal, 49(3), 544-560.

Coombs, J. E., \& Gilley, K. M. (2005). Stakeholder management as a predictor of CEO compensation: main effects and interactions with financial performance. Strategic Management Journal, 26(9), 827-840. doi: 10.1002/smj.476

Correa, E. A., \& Guimarães, T. A. (2006, setembro). Cultura de aprendizagem e desempenho em organizações. Validação de escala de medida e análise de suas relações. Anais do Encontro Nacional da Associação Nacional de Pós-Graduação e Pesquisa em Administração, Salvador, BA, Brasil, 30 .

Côté, S., \& Miners, C. T. H. (2006). Emotional intelligence, cognitive intelligence, and job performance. Administrative Science Quarterly, 51(1), 1-28. doi: 10.1097/ACM.0b013e3181eabe59

Creswell, J. W. (2002). Research design: qualitative, quantitative, and mixed methods approaches (2a ed.). London: Sage Publications. 
Crossland, C., \& Hambrick, D. C. (2007). How national systems differ in their constraints on corporate executives: a study of CEO effects in three countries. Strategic Management Journal, 28(8), 767-789. doi: 10.1002/smj.610

David, P., Bloom, M., \& Hillman, A. (2007). Investor activism, managerial responsiveness, and corporate social performance. Strategy Management Journal, 28(1), 91-100. doi: 10.1002/smj.571

Desarbo, W. S., Di Benedetto, A., Song, M., \& Sinha, I. (2005). Revisiting the miles and snow strategic framework: uncovering interrelationships between strategic types, capabilities, environmental uncertainties, and firm performance. Strategic Management Journal, 26(1), 4774. doi: $10.1002 / \mathrm{smj} .431$

Dias, A. T. (2006, setembro). Relações entre tamanho, diversificação, gestão de recursos, participação de mercado e desempenho organizacional de companhias brasileiras de capital aberto. Anais do Encontro Nacional da Associação Nacional de Pós-Graduação e Pesquisa em Administração, Salvador, BA, Brasil, 30.

Dias, A. T., \& Gonçalves, M. A. (2007, setembro). O papel mediador das estratégias corporativas nas relações entre ambiente e desempenho. Anais do Encontro Nacional da Associação Nacional de Pós-Graduação e Pesquisa em Administração, Rio de Janeiro, RJ, Brasil, 31.

Douma, S. W., George, R., \& Kabir, M. R. (2006). Foreign and domestic ownership, business groups, and firm performance: evidence from a large merging market. Strategic Management Journal, 27(7), 637-657. doi: 10.1002/smj.535

Ebben, J. J., \& Johnson, A. C. (2005). Efficiency, flexibility, or both? Evidence linking strategy to performance in small firms. Strategic Management Journal, 26(13), 1249-1259. doi: 10.1002/smj.503

Echols, A., \& Tsai, W. (2005) Niche and performance: the moderating role of network embeddedness. Strategic Management Journal, 26(3), 219-238. doi: 10.1002/smj.443

Eisenmann, T. R. (2006). Internet companies' growth strategies: determinants of investment intensity and long-term performance. Strategic Management Journal, 27(12), 1183-1204. doi: $10.1002 / \mathrm{smj} .567$

Ellis, A. P. J. (2006). System breakdown: the role of mental models and transitive memory in the relationship between acute stress and team performance. Academy of Management Journal, 49(3), 576-589.

Espinosa, J. A., Kraut, R. E., Slaughter, S. A., Lerch, J. F., \& Herbsleb, J. D. (2007). Familiarity, complexity, and team performance in geographically distributed software development. Organization Science, 18(4), 613-630. doi: 10.1287/orsc.1070.0297

Fang, Y., Wade, M., Delios, A., \& Beamish, P. (2007). International diversification, subsidiary performance and the mobility of knowledge resources. Strategic Management Journal, 28(10), 1053-1064. doi: 10.1002/smj.619

Faria, L. H. L., Brandão, M. M., \& Silva, V. G. (2005, setembro). Uma contribuição ao estudo da mensuração da performance através de suas componentes de variância: introduzindo a variável explanatória grupo estratégico. Anais do Encontro Nacional da Associação Nacional de PósGraduação e Pesquisa em Administração, Brasília, DF, Brasil, 29.

Fensterseifer, J. E., \& Wilk, E. O. (2005, setembro). Visão da firma baseada em recursos, clusters e performance: um estudo no setor vitivinícola do RS. Anais do Encontro Nacional da Associação Nacional de Pós-Graduação e Pesquisa em Administração, Brasília, DF, Brasil, 29. 
Fernandes, B. H. R., Fleury, M. T. L., \& Mills, J. F. (2006). Construindo um diálogo entre competências, recursos e desempenho organizacional. Revista de Administração de Empresas, 46(4), 48-65. doi: 10.1590/S0034-75902006000400006

Fischer, B. B. (2006, setembro). Relação entre estratégias de entrada em mercados estrangeiros e performance exportadora resultante em empresas brasileiras. Anais do Encontro Nacional da Associação Nacional de Pós-Graduação e Pesquisa em Administração, Salvador, BA, Brasil, 30.

Fontanelle, C., Hoeltgebaum, M., \& Silveira, A. (2006, setembro). A influência do perfil empreendedor dos franqueados no desempenho organizacional. Anais do Encontro Nacional da Associação Nacional de Pós-Graduação e Pesquisa em Administração, Salvador, BA, Brasil, 30.

Garrido, I. L. (2007, setembro). A relação entre orientação para mercado externo, performance internacional e estratégias de internacionalização. Anais do Encontro Nacional da Associação Nacional de Pós-Graduação e Pesquisa em Administração, Rio de Janeiro, RJ, Brasil, 31.

George, G. (2005). Slack resources and the performance of privately held firms. Academy of Management Journal, 48(4), 661-676.

Glunk, U., \& Wilderom, C. P. M. (1999). Organizational effectiveness = corporate performance? Why and how two research approaches need to be merged. In M. P. Cunha \& C. A. Marques (Eds.), Readings in organization science: organizational change in a changing context (pp. 155-181). Lisboa: ISPA.

Goerzen, A., \& Beamish, P. (2005). The effect of alliance network diversity on multinational enterprise performance. Strategic Management Journal, 26(4), 333-354. doi: 10.1002/smj.447

Goldszmidt, R. G. B., Brito, L. A. L., \& Vasconcelos, F. C. (2007a). O efeito país sobre o desempenho da firma: uma abordagem multinível. Revista de Administração de Empresas, 47(1), 12-23. doi: 10.1590/S0034-75902007000100003

Goldszmidt, R. G. B., Brito, L. A. L., \& Vasconcelos, F. C. (2007b, setembro). Países ou empresas competitivas? Uma análise exploratória da relação entre desempenho das firmas e competitividade das nações. Anais do Encontro Nacional da Associação Nacional de PósGraduação e Pesquisa em Administração, Rio de Janeiro, RJ, Brasil, 31.

Gonçalves, A. R., \& Quintella, R. H. (2005, setembro). Contribuição dos fatores internos e externos para o desempenho das empresas brasileiras e sua evolução na última década. Anais do Encontro Nacional da Associação Nacional de Pós-Graduação e Pesquisa em Administração, Brasília, DF, Brasil, 29.

Gonçalves, A. R., \& Quintella, R. H. (2006). The role of internal and external factors in the performance companiens and its evolution between 1990 and 2003 [Edição Especial]. Revista de Administração Contemporânea, 10, 117-136. doi: 10.1590/S1415-65552006000500007

Gonçalves, C. A., Gosling, M., \& Lanna, M. A. M. F. (2007, setembro). A influência da qualidade das incubadoras e da formulação estratégica dos empreendimentos incubados no desempenho empresarial. Anais do Encontro Nacional da Associação Nacional de Pós-Graduação e Pesquisa em Administração, Rio de Janeiro, RJ, Brasil, 31.

Gong, Y., Shenkar, O., Luo, Y., \& Nyaw, M. (2007). Do multiple parents help or hinder international joint venture performance? The mediating roles of contract completeness and partner cooperation. Strategic Management Journal, 28(10), 1021-1034. doi: 10.1002/smj.626

Goold, M., Young, D., \& Collis, D. (2007). The size, structure, and performance of corporate headquarters. Strategic Management Journal, 28(4), 383-405. doi: 10.1002/smj.595 
Greenwood, R., Li, S. X., Prakash, R., \& Deephouse, D. L. (2005). Reputation, diversification, and organizational explanations of performance in professional service firms. Organization Science, 16(6), 661-673. doi: 10.1287/orsc.1050.0159

Grzebieluckas, C., Alberton, A., Marcon, R., \& Kruger, R. E. (2006, setembro). Exploring stakeholder contracting effects on firm performance. Anais do Encontro Nacional da Associação Nacional de Pós-Graduação e Pesquisa em Administração, Salvador, BA, Brasil, 30.

Grzebieluckas, C., Marcon, R., \& Alberton, A. (2007, setembro). A estratégia de diversificação e sua influência na performance: uma análise empírica em companhias abertas no Brasil. Anais do Encontro Nacional da Associação Nacional de Pós-Graduação e Pesquisa em Administração, Rio de Janeiro, RJ, Brasil, 31.

Gulati, R., \& Sytch, M. (2007). Dependence asymmetry and joint dependence in interorganizational relationships: effects of embeddedness on a manufacturer's performance in procurement relationships. Administrative Science Quarterly, 52(1), 32-69.

Haas, M. R., \& Hansen, M. T. (2005). When using knowledge can hurt performance: the value of organizational capabilities in a management consulting company. Strategic Management Journal, 26(1), 1-24. doi: 10.1002/smj.429

Haleblian, J., Kim, Y., \& Rajagopalan, N. (2006). The influence of acquisition experience and performance on acquisition behavior: evidence from the U.S. commercial banking industry. Academy of Management Journal, 49(2), 357-370.

Harris, J., \& Bromiley, P. (2007). Incentives to cheat: the influence of executive compensation and firm performance on financial misrepresentation. Organization Science, 18(3), 350-367. doi: 10.1287/orsc.1060.0241

Hayward, M. L. A., \& Shimizu, K. (2006). De-commitment to losing strategic action: evidence from the divestiture of poorly performing acquisitions. Strategic Management Journal, 27(6), 541557. doi: 10.1002/smj.526

Henderson, A. D., Miller, D., \& Hambrick, D. C. (2006). How quickly do CEOs become obsolete? Industry dynamism, CEO tenure, and company performance. Strategic Management Journal, 27(5), 447-460. doi: 10.1002/smj.524

Hoang, H., \& Rothaermel, F. T. (2005). The effect of general and partner-specific alliance experience on joint R\&D project performance. Academy of Management Journal, 48(2), 332-345.

Hough, J. R. (2006). Business segment performance redux: a multilevel approach. Strategic Management Journal, 27(1), 45-61. doi: 10.1002/smj.498

Hult, G. T. M., Ketchen, D. M., Jr., \& Arrfelt, M. (2007). Strategic supply chain management: improving performance through a culture of competitiveness and knowledge management. Strategic Management Journal, 28(10), 1035-1052. doi: 10.1002/smj.627

Hunter, L. W., \& Thatcher, S. M. B. (2007). Feeling the heat: effects of stress, commitment, and job experience on job performance. Academy of Management Journal, 50(4), 953-968.

Joshi, A., Liao, H., \& Jackson, S. E. (2006). Cross-level effects of workplace diversity on sales performance and pay. Academy of Management Journal, 49(3), 459-481.

Kacmar, K. M., Andrews, M. C., Rooy, D. L. van, \& Steilberg, R. C. (2006). Sure everyone can be replaced ... but at what cost? Turnover as a predictor of unit-level performance. Academy of Management Journal, 49(1), 133-144. 
Karaevli, A. (2007). Performance consequences of new CEO "outsiderness": moderating effects of pre- and post-succession contexts. Strategic Management Journal, 28(7), 681-706. doi: $10.1002 / \mathrm{smj} .589$

Kassinis, G., \& Vafeas, N. (2006). Stakeholder pressures and environmental performance. Academy of Management Journal, 49(1), 145-159. doi: 10.2307/20159751

Katsikeas, C. S., Leonidou, L. C., \& Morgan, N. A. (2000). Firm-level export performance assessment: review, evaluation, and development. Journal of the Academy of Marketing Science, 28(4), 493-511. doi: 10.1177/0092070300284003

Katsikeas, C. S., Samiee, S., \& Theodosiou, M. (2006). Strategy fit and performance consequences of international marketing standardization. Strategic Management Journal, 27(9), 867-890. doi: $10.1002 / \mathrm{smj} .549$

Klein, K. J., \& Kozlowski, S. W. J. (Eds.). (2000). Multilevel theory, research, and methods in organizations: foundations, extensions, and new directions. San Francisco, CA: Jossey-Bass.

Klotzle, M. C., \& Thomé, C. C. (2006). Fatores associados ao desempenho exportador de micros, pequenas e médias empresas brasileiras. Revista de Administração da USP, 41(3), 339-346.

Kor, Y., \& Leblebici, H. (2005). How do interdependencies among human-capital deployment, development, and diversification strategies affect firms' financial performance? Strategic Management Journal, 26(10), 967-985. doi: 10.1002/smj.485

Kor, Y. Y., \& Mahoney, J. T. (2005). How dynamics, management, and governance of resource deployments influence firm-level performance. Strategic Management Journal, 26(5), 489-496. doi: $10.1002 / \mathrm{smj} .459$

Krishnan, R., Martin, X., \& Noordhaven, N. G. (2006). When does trust matter to alliance performance? Academy of Management Journal, 49(5), 894-917. doi: 10.2307/20159808

Kroll, M., Walters, B. A., \& Le, S. A. (2007). The impact of board composition and top management team ownership structure on post-IPO performance in young entrepreneurial firms. Academy of Management Journal, 50(5), 1198-1216.

Labianca, G., Moon, H., \& Watt, I. (2005). When is an hour not sixty minutes? Deadlines, temporal schemas, and individual and group task performance. Academy of Management Journal, 48(4), 677-694. doi: 10.2307/20159686

Laursen, K., \& Salter, A. (2006). Open for innovation: the role of openness in explaining innovative performance among UK manufacturing firms. Strategic Management Journal, 27(2), 131-150. doi: $10.1002 / \mathrm{smj} .507$

Lavie, D. (2007). Alliance portfolios and firm performance: a study of value creation and appropriation in the U.S. software industry. Strategic Management Journal, 28(12), 1187-1212. doi: $10.1002 /$ smj.637

Lavie, D., Lechner, C., \& Harbir, S. (2007). The performance implications of timing of entry and involvement in multipartner alliances. Academy of Management Journal, 50(3), 578-604. doi: $10.2307 / 20159874$

Lazzarini, S. G. (2007). The impact of membership in competing alliance constellations: evidence on the operational performance of global airlines. Strategic Management Journal, 28(4), 345-367. doi: 10.1002/smj.587

Leana, C., \& Pil, F. (2006). Social capital and organizational performance: evidence from urban public schools. Organization Science, 17(3), 353-366. doi: 10.1287/orsc.1060.0191 
Leão, D. A. F. S. (2005, setembro). Coopetição: tipologia e impactos no desempenho das empresas da indústria de cachaça de Alambique do Estado de Minas Gerais. Anais do Encontro Nacional da Associação Nacional de Pós-Graduação e Pesquisa em Administração, Brasília, DF, Brasil, 29.

Lewin, A. Y., \& Minton, J. W. (1986). Determining organizational effectiveness: another look, and an agenda for research. Management Science, 32(5), 514-538. doi: 10.1287/mnsc.32.5.514

Li, H., \& Zhang, Y. (2007). The role of managers' political networking and functional experience in new venture performance: evidence from China's transition economy. Strategic Management Journal, 28(8), 791-804. doi: 10.1002/smj.605

Love, G. E., \& Nohria, N. (2005). Reducing slack: the performance consequences of downsizing by large industrial firms, 1977-93. Strategic Management Journal, 26(12), 1087-1108. doi: 10.1002/smj.487

Luo, X., \& Chung, C. (2005). Keeping it all in the family: the role of particularistic relationships in business group performance during institutional transition. Administrative Science Quarterly, 50(3), 404-439.

Macher, J. T., \& Boerner, C. S. (2006). Experience and scale and scope economies: trade-offs and performance in development. Strategic Management Journal, 27(9), 845-865. doi: 10.1002/smj

Madsen, T. (1998). Managerial judgment and export performance. Journal of International Marketing, 6(3), 82-93.

Makri, M., Lane, P. J., \& Gomez-Mejia, L. (2006). CEO incentives, innovation, and performance in technology-intensive firms. Strategic Management Journal, 27(11), 1057-1080. doi: $10.1002 / \mathrm{smj} .560$

Mathieu, J. E., \& Schulze, W. (2006). The influence of team knowledge and formal plans on episodic team process-performance relationships. Academy of Management Journal, 49(3), 605-619.

Matthyssens, P., \& Pauwels, P. (1996). Assessing export performance measurement. In S. T. Cavusgil \& C. Axinn (Eds.), Advances in International Marketing (Vol. 8, pp. 85-114). Greenwich, CT: JAI Press Inc.

Maurer, I., \& Ebers, M. (2006). Dynamics of social capital and their performance implications: lessons from biotechnology start-ups. Administrative Science Quarterly, 51(2), 262-292.

Mayer, K. J., \& Nickerson, J. A. (2005). Antecedents and performance implications of contracting for knowledge workers: evidence from information technology services. Organization Science, 16(3), 225-242. doi: 10.1287/orsc.1050.0125

Mayer, R. C., \& Gavin, M. B. (2005). Trust in management and performance: who minds the shop while the employees watch the boss? Academy of Management Journal, 48(5), 874-888. doi: $10.2307 / 20159703$

McNamara, G., Aime, F., \& Vaaler, P. (2005). Is performance driven by industry- or firm-specific factors? A response to Hawawini, Subramanian, and Verdin. Strategic Management Journal, 26(11), 1075-1081. doi: 10.1002/smj.496

Mehra, A., Dixon, A., Brass, D. J., \& Robertson, B. (2006). The social network ties of group leaders: Implications for group performance and leader reputation. Organization Science, 17(1), 64-79. doi: 10.1287/orsc.1050.0158

Mello, R. B., \& Marcon, R. (2006). Heterogeneidade do desempenho de empresas em ambientes turbulentos. Revista de Administração de Empresas, 46(2), 1-16. doi: 10.1590/S003475902006000200004 
Miele, M., \& Zylbersztajn, D. (2005). Coordenação e desempenho da transação entre viticultores e vinícolas na serra Gaúcha. Revista de Administração da USP, 40(4), 330-341.

Miller, S. R., \& Eden, L. (2006). Local density and foreign subsidiary performance. Academy of Management Journal, 49(2), 341-355. doi: 10.2307/20159767

Moraes, E. A. (2006, setembro). Análise do desempenho de empresas utilizando modelos de componentes de variância. Anais do Encontro Nacional da Associação Nacional de PósGraduação e Pesquisa em Administração, Salvador, BA, Brasil, 30.

Moran, P. (2005). Structural vs. relational embeddedness: social capital and managerial performance. Strategic Management Journal, 26(12), 1129-1151. doi: 10.1002/smj.486

Nasser-Carvalho, L. F. (2005, setembro). Cognição em organizações: complexidade cognitiva e seus impactos no desempenho organizacional. Anais do Encontro Nacional da Associação Nacional de Pós-Graduação e Pesquisa em Administração, Brasília, DF, Brasil, 29.

Omaki, E. T. (2005, setembro). Recursos intangíveis e desempenho em grandes empresas brasileiras: avaliações dos recursos intangíveis como estimador de medidas de desempenho financeiras. Anais do Encontro Nacional da Associação Nacional de Pós-Graduação e Pesquisa em Administração, Brasília, DF, Brasil, 29.

Park, K. M. (2007). Antecedents of convergence and divergence in strategic positioning: the effects of performance and aspiration on the direction of strategic change. Organization Science, 18(3), 386-402. doi: 10.1287/orsc.1060.0240

Payne, G. T. (2006) Examining configurations and firm performance in a suboptimal equifinality context. Organization Science, 17(6), 756-770. doi: 10.1287/orsc.1060.0203

Pehrsson, A. (2006). Business relatedness and performance: a study of managerial perceptions. Strategic Management Journal, 27(3), 265-282. doi: 10.1002/smj.516

Pereira, B. A. D. (2005, setembro). Identificação de fatores determinantes do desempenho das empresas inseridas em redes horizontais. Anais do Encontro Nacional da Associação Nacional de Pós-Graduação e Pesquisa em Administração, Brasília, DF, Brasil, 29.

Perin, M. G., Sampaio, C. H., \& Hooley, G. J. (2007). Impacto dos recursos da empresa na performance de inovação. Revista de Administração de Empresas, 47(1), 46-58. doi: 10.1590/S0034-75902007000100005

Pilati, R., \& Borgesandrade, J. (2005, setembro). Comprometimento no trabalho, motivação e estratégias de aplicação como preditores de efetividade de treinamento. Anais do Encontro Nacional da Associação Nacional de Pós-Graduação e Pesquisa em Administração, Brasília, DF, Brasil, 29.

Porath, C. L., \& Erez, A. (2007). Does rudeness really matter? The effects of rudeness on task performance and helpfulness. Academy of Management Journal, 50(5), 1181-1197. doi: $10.2307 / 20159919$

Quigley, N. R., Tesluk, P. E., Locke, E. A., \& Bartol, K. M. (2007). A multilevel investigation of the motivational mechanisms underlying knowledge sharing and performance. Organization Science, 18(1), 71-88. doi: 10.1287/orsc.1060.0223

Raver, J. L., \& Gelfand, M. J. (2005). Beyond the individual victim: linking sexual harassment, team processes, and team performance. Academy of Management Journal, 48(3), 387-400. 
Richard, P. J., Devinney, T. M., Yip, G. S., \& Johnson, G. (2009). Measuring organizational performance: towards methodological best practices. Journal of Management, 35(3), 718-804. doi: $10.1177 / 0149206308330560$

Rogers, P., Mendes-da-Silva, W., \& Paula, G. M. (2005, setembro). Efeitos da estratégia de diversificação no desempenho das indústrias brasileiras: um estudo de companhias de capital aberto no período de 1997 a 2001. Anais do Encontro Nacional da Associação Nacional de PósGraduação e Pesquisa em Administração, Brasília, DF, Brasil, 29.

Rothaermel, F., \& Hill, C. W. (2005). Technological discontinuities and complementary assets: a longitudinal study of industry and firm performance. Organization Science, 16(1), 52-70. doi: 10.1287/orsc. 1040.0100

Rothaermel, F. T., Hitt, M. A., \& Jobe, L. A. (2006). Balancing vertical integration and strategic outsourcing: effects on product portfolios, new product success, and firm performance. Strategic Management Journal, 27(11), 1033-1056. doi: 10.1002/smj.559

Russo, G., \& Macedo-Soares, T. D. L. E. van (2007, setembro). Benefícios essenciais das alianças estratégicas no modelo de co-opetição entre empresas aéreas: resultados de análise empírica e proposta preliminar de indicadores de desempenho. Anais do Encontro Nacional da Associação Nacional de Pós-Graduação e Pesquisa em Administração, Rio de Janeiro, RJ, Brasil, 31.

Russo, M. V., \& Harrison, N. S. (2005). Organizational design and environmental performance: clues from the electronics industry. Academy of Management Journal, 48(4), 582-593. doi: $10.2307 / 20159680$

Salles, H. K., \& Dellagnelo, E. H. L. (2005, setembro). A estruturação de organizações ambientalistas do terceiro setor e a relação com o modelo de avaliação de desempenho de suas fontes financiadoras. Anais do Encontro Nacional da Associação Nacional de Pós-Graduação e Pesquisa em Administração, Brasília, DF, Brasil, 29.

Sampson, R. C. (2007). R\&D alliances and firm performance. The impact of technological diversity and alliance organization on innovation. Academy of Management Journal, 50(2), 364-386. doi: $10.2307 / 20159859$

Sanders, W. G., \& Hambrick, D. C. (2007). Swinging for the fences: the effects of CEO stock options on company risk taking and performance. Academy of Management Journal, 50(5), 1055-1078.

Scheible, A. C. F., \& Bastos, A. V. B. (2005, setembro). Práticas de gestão democrática como mediador da relação entre comprometimento e desempenho. Anais do Encontro Nacional da Associação Nacional de Pós-Graduação e Pesquisa em Administração, Brasília, DF, Brasil, 29.

Scheible, A. C. F., Batos, A. V. B., \& Rodrigues, A. C. A. (2007, setembro). Comprometimento e entrincheiramento: integrar ou reconstruir? Uma exploração das relações entre estes construtos à luz do desempenho. Anais do Encontro Nacional da Associação Nacional de Pós-Graduação e Pesquisa em Administração, Rio de Janeiro, RJ, Brasil, 31.

Schmidt, F. H. (2006, setembro). O impacto das estratégias colaborativas na indústria de transporte aéreo. Anais do Encontro Nacional da Associação Nacional de Pós-Graduação e Pesquisa em Administração, Salvador, BA, Brasil, 30.

Shaw, J. D., Duffy, M. K., Johnson, J. J., \& Lockhart, D. (2005). Turnover, social capital losses, and performance. Academy of Management Journal, 48(4), 594-606.

Shaw, J. D., Gupta, N., \& Delery, J. F. (2005). Alternative conceptualizations of the relationship between voluntary turnover and organizational performance. Academy of Management Journal, 48(1), 50-68. 
Shipilov, A. V. (2006). Network strategies and performance of Canadian investment banks. Academy of Management Journal, 49(3), 590-604. doi: 10.2307/20159783

Short, J. C., Ketchen, D. J., Palmer, T. B., \& Hult, T. (2007). Firm, strategic group, and industry influences on performance. Strategic Management Journal, 28(2), 147-167. doi: 10.1002/smj.574

Siegel, P., \& Hambrick, D. C. (2005). Pay disparities within top management groups: evidence of harmful effects on performance of high-technology firms. Organization Science, 16(3), 259274. doi: $10.1287 /$ orsc. 1050.0128

Silva, J. F., Motta, P. C. M., \& Costa, L. S. V. (2007). Coalinhamento entre estratégias competitivas e colaborativas e desempenho de empresas. Revista de Administração de Empresas, 47(4), 26-45. doi: 10.1590/S0034-75902007000400004

Sine, W. D., Mitsuhashi, H., \& Kirsch, D. A. (2006). Revisiting Burns and Stalker: formal structure and new venture performance in emerging economic sectors. Academy of Management Journal, 49(1), 121-132. doi: 10.2307/20159749

Soares, F. A., Abreu, M. C. S., \& Sampaio, J. (2006, setembro). A relação entre a evidenciação ambiental e a performance econômica na indústria siderúrgica brasileira. Anais do Encontro Nacional da Associação Nacional de Pós-Graduação e Pesquisa em Administração, Salvador, BA, Brasil, 30 .

Sorenson, O., McEvily, S., Ren, C. R., \& Roy, R. (2006). Niche width revisited: organizational scope, behavior and performance. Strategic Management Journal, 27(10), 915-936. doi: 10.1002/smj

Souto, S. O., \& Rego, J. A. (2006, setembro). Espiritualidade nas organizações, positividade e desempenho. Anais do Encontro Nacional da Associação Nacional de Pós-Graduação e Pesquisa em Administração, Salvador, BA, Brasil, 30.

Souza, A. F., Neto, \& Cordeiro, A. T. (2006, setembro). Orientação empreendedora, incubação e redes de empresas vis-à-vis competências mercadológicas e desempenho: um estudo em empresas de base tecnológica. Anais do Encontro Nacional da Associação Nacional de Pós-Graduação e Pesquisa em Administração, Salvador, BA, Brasil, 30.

Spers, R. G., \& Wright, J. T. C. (2006, setembro). Mercado de bens populares no Brasil: desempenho e estratégia das empresas. Anais do Encontro Nacional da Associação Nacional de PósGraduação e Pesquisa em Administração, Salvador, BA, Brasil, 30.

Srivastava, A., Bartol, K. M., \& Locke, E. A. (2006). Empowering leadership in management teams: Effects on knowledge sharing, efficacy, and performance. Academy of Management Journal, 49(6), 1239-1251.

Steers, R. M. (1975). Problems in the measurement of organizational effectiveness. Administrative Science Quarterly, 20(4), 546-558.

Sun, L., Aryee, S., \& Law, K. S. (2007). High performance human resource practices, citizenship behavior, and organizational performance: a relational perspective. Academy of Management Journal, 50(3), 558-577.

Tanriverdi, H., \& Venkatraman, N. (2005). Knowledge, relatedness and performance of multibusiness. Firms. Strategic Management Journal, 26(2), 97-119. doi: 10.1002/smj.435

Thach, S., \& Axinn, C. (1994). Redefining export success: not which measures, but why measure. Anais do Consortium for International Marketing Research Conference, Rio de Janeiro, RJ, Brasil. 
Van der Vegt, G. S., \& Bunderson, J. S. (2005). Learning and performance in multidisciplinary teams: The importance of collective team identification. Academy of Management Journal, 48(3), 532547. doi: $10.2307 / 20159674$

Vasconcelos, E. M. (2002). Complexidade e pesquisa interdisciplinar: epistemologia e metodologia operativa. Petrópolis, RJ: Vozes.

Veit, M. R., \& Gonçalves, C., Filho (2007, setembro). Mensuração do perfil do potencial empreendedor e seu impacto no desempenho das pequenas empresas. Anais do Encontro Nacional da Associação Nacional de Pós-Graduação e Pesquisa em Administração, Rio de Janeiro, RJ, Brasil, 31.

Vera, D., \& Crossan, M. (2005). Improvisation and innovative performance in teams. Organization Science, 16(3), 203-224. doi: 10.1287/orsc.1050.0126

Von Nordenflycht, A. (2007). Is public ownership bad for professional service firms? Ad agency ownership, performance, and creativity. Academy of Management Journal, 50(2), 429-445.

Voss, Z. G., Cable, D. M., \& Voss, G. B. (2006). Organizational identity and firm performance: what happens when leaders disagree about "who we are?" Organization Science, 17(6), 741-755. doi: 10.1287/orsc.1060.0218

Wade, J. B., Porac, J. F., Pollock, T. G., \& Graffin, S. D. (2006). The burden of celebrity: the impact of CEO certification contests on CEO pay and performance. Academy of Management Journal, 49(4), 643-660.

Wang, H., Law, K. S., Hackett, R. D., Wang, D., \& Chen, Z. (2005). Leader-member exchange as a mediator of the relationship between transformational leadership behavior and followers' performance and organizational citizenship behavior. Academy of Management Journal, 48(3), 420-432. doi: 10.2307/20159668

Westphal, J. D., \& Bednar, M. (2005). Pluralistic ignorance in corporate boards and strategic persistence in response to low firm performance. Administrative Science Quarterly, 50(2), 262298.

Yakubovich, V., \& Lup, D. (2006). Stages of the recruitment process and the referrer? performance effect. Organization Science, 17(6), 710-723. doi: 10.1287/orsc.1060.0214

Zaheer, A., \& Bell, G. G. (2005). Benefiting from network position: firm capabilities, structural holes and performance. Strategic Management Journal, 26(9), 809-826. doi: 10.1002/smj.482

Zellmer-Bruhn, M., \& Gibson, C. (2006). Multinational organization context: implications for team learning and performance. Academy of Management Journal, 49(3), 501-518. 